\title{
On the impulsive controllability and bifurcation of a predator-pest model of IPM
}

\author{
Hong Zhang ${ }^{\mathrm{a}, *}$, Paul Georgescu ${ }^{\mathrm{b}}$, Lansun Chen ${ }^{\mathrm{c}}$ \\ ${ }^{a}$ Department of Mathematics, Jiangsu University, Zhenjiang, Jiangsu 212013, PR China \\ b Department of Mathematics, "Gh. Asachi” Technical University of Iaşi, Bd. Copou 11, 700506 Iaşi, Romania \\ ${ }^{\mathrm{c}}$ Institute of Mathematics, Academia Sinica, Beijing 100080, PR China
}

Received 12 October 2007; received in revised form 9 March 2008; accepted 19 March 2008

\begin{abstract}
From a practical point of view, the most efficient strategy for pest control is to combine an array of techniques to control the wide variety of potential pests that may threaten crops in an approach known as integrated pest management (IPM). In this paper, we propose a predator-prey (pest) model of IPM in which pests are impulsively controlled by means of spraying pesticides (the chemical control) and releasing natural predators (the biological control). It is assumed that the biological and chemical control are used with the same periodicity, but not simultaneously. The functional response of the predator is allowed to be predator-dependent, in the form of a Beddington-DeAngelis functional response, rather than to have a perhaps more classical prey-only dependence. The local and global stability of the pest-eradication periodic solution, as well as the permanence of the system, are obtained under integral conditions which are shown to have biological significance. In a certain limiting case, it is shown that a nontrivial periodic solution emerges via a supercritical bifurcation. Finally, our findings are confirmed by means of numerical simulations.
\end{abstract}

(C) 2008 Elsevier Ireland Ltd. All rights reserved.

Keywords: Beddington-DeAngelis functional response; Impulsive controls; Stability analysis; Pest-eradication periodic solution; Permanence; Fixed point approach; Supercritical bifurcation; Economic threshold

\section{Introduction}

Plants, insects, mildew, rodents, bacteria and other organisms are a natural part of our environment. Some of these organisms can be beneficial to people in many ways while some others are pests which produce less desirable effects. Our apartments and houses are often hosts to common pests, for example, cockroaches, fleas, termites, ants, mice, rats and mold. Weeds, hornworms, aphids and grubs can be a nuisance outdoors when they get into lawns, flowers, yards, vegetable gardens, or fruit trees. Pests can also be a health catastrophe for all human beings and pets alike. It is easy to understand why one may need and want to control them.

For many pests, total elimination is almost impossible or at least unfeasible due to a variety of causes. In the meantime, they may be kept below certain economically and perceptually significant levels. In this regard, knowingly using the available options is the key to pest control. Among the methods available to fulfill the goal of successfully controlling and containing the spread of pests we mention pest prevention, non-chemical pest controls (Freedman, 1976, Ferron, 1981; Luff, 1983; Debach and Rosen, 1991), defined as the use of a suitably chosen living organism, referred as the biocontrol agent, to control the pest, and chemical pesticides that kill the pest directly, usually by exposing it to lethal substances or unsuitable environmental conditions, reduce the reproductive potential of a pest population, often by modifying its environment (biotic or abiotic) or by restricting its movement, and modify the pest's behavior to make it less troublesome (attract it, repel it, confuse it, exclude it and mislead it).

The most effective strategy for pest control is to combine an array of methods in an approach known as integrated pest management (IPM) that emphasizes reducing pests to tolerable levels, with little cost to the grower and minimal possible hazard to people, property

\footnotetext{
* Corresponding author.

E-mail addresses: hongzhang@ujs.edu.cn, cnczzhanghong@163.com (H. Zhang), vpgeo@go.com (P. Georgescu), 1schen@amss.ac.cn (L.Chen).
} 
and environment. The concept of IPM was introduced in the late 1950s (Stern et al., 1959) and was widely practised during the 1970s and 1980s (Van den Bosch, 1978).

Recently, many papers have been devoted to the analysis of mathematical models describing IPM strategies. See, for instance Liu et al. (2004); Zhang et al. (2004); Tang and Chen (2004); Liu et al. (2005); Zhang et al. (2007a, b); Georgescu et al. (2008). All these prey-predator (target pest-its natural enemy) models do, however, assume that predators do not interfere with one another and consequently their functional and numerical responses depend upon the size of the prey population only. That is, following the terminology given in (Arditi and Ginzburg, 1989; Huisman and DeBoer, 1997), papers on the mathematical modeling of IPM strategies have focused on prey-dependent models. However, a significant shortcoming of prey-dependent models is that they do not account for the effects of interference or cooperation between predators, which is why they have been challenged by the mathematical modeling community (Abrams and Ginzburg, 2000; Arditi and Ginzburg, 1989; Kuang and Beretta, 1998; Gutierrez, 1992).

There is much significant evidence to suggest that predator dependence in the functional response occurs quite frequently in laboratory and within natural systems (see, for instance, Jost and Ellner, 2000 or Skalski and Gilliam, 2001) and due to large numbers of experiments and observations, the models with predator-dependent functional response stand as reasonable alternatives to the models with prey-dependent functional response. Arditi and Ginzburg (1989) first proposed and investigated the following ratiodependent (that is, a model in which the functional response of the predator depends upon the prey-to-predator ratio) predator-prey model:

$$
\left\{\begin{array}{l}
N^{\prime}=N(a-b N)-\frac{c N P}{m P+N}, \\
P^{\prime}=-\mathrm{d} P+\frac{f N P}{m P+N},
\end{array}\right.
$$

where $N$ denotes the size of the prey class and $P$ denotes the size of the predator class. Note that the above model is a result of replacing the Holling type II prey-dependent functional response $N /(m+N)$ by a ratio-dependent one $(N / P) /(m+N / P)$.

It is, however, to be noted that, although they do succeed in modeling the effects of interference between predators, ratio-dependent models do not escape criticism. Specifically, an issue which affects ratio-dependent models is the "low-density problem", that is, the fact that the attack ratio of the predators becomes infinite when the size of the predator class tends to 0 .

The Beddington-DeAngelis functional response

$$
F(N, P)=\frac{\alpha N}{a+b N+c P}
$$

was originally introduced by Beddington (1975) and DeAngelis et al. (1975), independently, and actually for different reasons. This predator-dependent functional response keeps certain qualitative features of the ratio-dependent functional responses, but stays away from the "low-densities problem".

Analyzing a wide range of predator-prey interactions and predator functional responses, Skalski and Gilliam (2001) pointed out that predator-dependent functional response provide a more accurate description and prediction of the practical outcomes, with the Beddington-DeAngelis functional response topping the list in some cases, although it has also been found that no single functional response can accommodate all the data sets.

On the other hand, as far as chemical controls are concerned, an acceptable dose of synthetic pesticides are used in IPM strategies when deemed an absolute necessity and generally have the collateral effect of removing small proportions of the natural enemies of the pests as well. To compensate pesticides-induced mortality of natural enemies, an appropriate amount of predators should consequently be introduced into the ecosystem. To account for the fact that farmers cannot spray pesticides and release natural enemies of the pests continuously, we have chosen to use impulsive controls in our mathematical model. This particular choice is, in our opinion, justified since for certain pesticides the effect follows shortly after application and also since the size of the natural enemies population grows immediately after each release. Therefore, such changes can be modeled as immediate jumps in the population sizes. In this regard, a general account of the theory of impulsive ordinary differential equations can be found in Bainov and Simeonov (1993).

Supposing that the control by means of releasing natural enemies of the pests is cheap, effective, permanent and does not disrupt the ecosystem, from a practical point of view, in IPM programs, the goal is to answer the following crucial questions: How many natural enemies should one release? What proportion of pests needs to be removed each time the pesticides are sprayed? How does one estimate the appropriate period for the impulsive controls according to the parameters of the system in order to keep the amount of the target pest class below the economic threshold (ET) which indicates the maximally admissible pest densities (numbers of preys per unit area)?

As motivated by the above-mentioned brief literature survey, the aim of this paper is to construct an impulsive differential model of predator-pest interaction incorporating a Beddington-DeAngelis functional response (Beddington, 1975; DeAngelis et al., 1975; Liu and Beretta, 2006), an impulsive biological control consisting in the periodic release of predators (natural enemies of pests) in a constant amount and an impulsive chemical control consisting in periodic pesticide spraying, with a periodicity similar to that of the biological controls, but avoiding the simultaneous use of the controls, and to investigate the dynamics of this system. We thereby 
suggest an impulsive system which includes two trophic levels, prey and predator, to model the target pest and its natural enemies, respectively.

This paper is organized in the following manner. We propose the model to be studied in Section 2 and give certain preliminary results in Section 3, together with our basic notations. In Section 4, the local and global stability of the prey (pest)-eradication periodic solution is studied by means of linearized Floquet stability and by means of comparison techniques, respectively. Section 5 is dedicated to establishing sufficient conditions for the permanence of the system. By employing an operator theoretic approach which reduces the existence of the nontrivial periodic solutions to a fixed point problem, it is then shown in Section 6 that once a threshold condition is reached, the trivial periodic solution loses its stability and a nontrivial periodic solution appears via a supercritical bifurcation. A number of more technical computations used to establish the results presented in this section are deferred to an Appendix A. Numerical simulations which confirm our theoretical findings are given in Section 7. Finally, a discussion of the ecological implications of our results is provided and some future research directions are outlined.

\section{The model}

The abundance and interaction of prey and predator populations (pests and their natural enemies, that is) may be expressed in terms of their biomass per spatial unit. In this regard, let $x(t), y(t)$ be the biomass per spatial unit of the prey and predator, respectively. The predators are assumed to be characterized by a Beddington-DeAngelis functional response, that is, by a response $F$ of the form

$$
F(x(t), y(t))=\frac{b x(t)}{A+k_{1} x(t)+k_{2} y(t)},
$$

depending not only on the prey biomass density $x(t)$ but also on the predator biomass density $y(t)$, and satisfying a few assumptions which will be outlined below. In view of the assumption above, the predators do not exhibit the "low-densities problem" since $A>0$. For convenience, it is supposed that in the absence of predation the dynamics of the prey population follows a logistic growth law with intrinsic growth rate 1 and carrying capacity $K$.

The coefficients $b$ and $k_{1}$ are positive constants that represent the effects of capture rate and handling time, respectively, on the feeding rate; $\beta$ is the birth rate of the predator; and $k_{2} \geq 0$ is a constant describing the magnitude of interference among predators; $w$ denotes the death rate of the predator population.

It is assumed that predators are bred and subsequently released in an impulsive and periodic fashion of period $T$, in a fixed amount $\mu$ each time. It is also assumed that pesticides are sprayed in an impulsive and periodic fashion, with the same period as the action of releasing predators, but at different moments. As a result of pesticide spraying, fixed proportions $p_{1}$ and $p_{2}$ of the prey and predator biomass are degraded each time.

On the basis of the above assumptions, we can formulate the model to be studied in the following way:

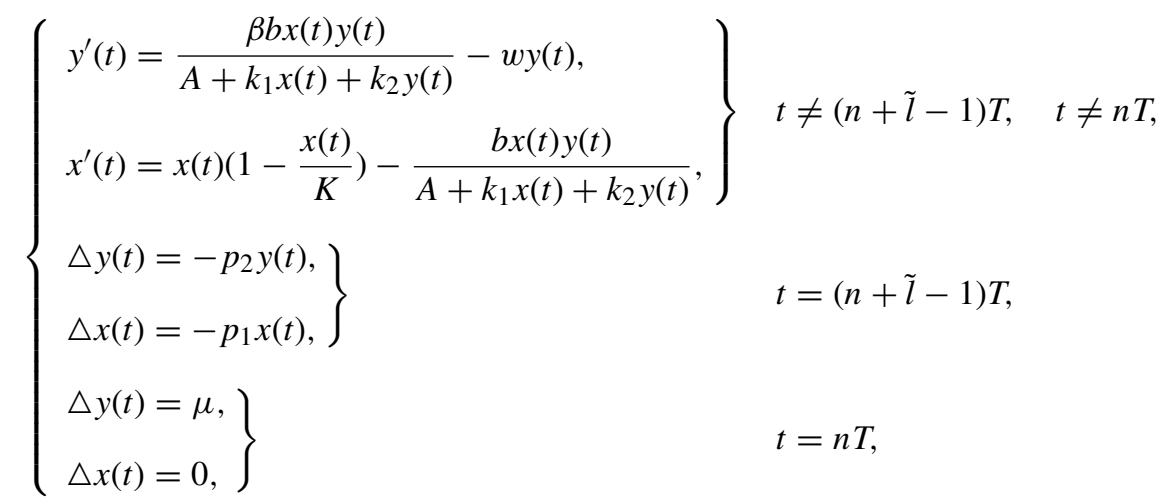

Here, $0<\tilde{l}<1$ is used to describe the intervals of time between the pulsed use of controls, of length $\tilde{l} T$ and $(1-\tilde{l}) T$. By using techniques similar to those employed in Liu et al. (2005), Zhang et al. (2007a), that is, linearized Floquet theory of impulsively perturbed systems of ordinary differential equations and comparison methods (see Lakshmikantham et al., 1989; Bainov and Simeonov, 1993 for details related to these theoretical devices), we establish local and global stability results for the pest-eradication periodic solution under appropriate conditions. Note that, due to the supply of predators by means of using the biological control, our controlled system does not exhibit the domino effect, characteristic to the unperturbed predator-prey system, that is, if the species at lowest trophic level dies out then all the species at higher trophic levels die out as well. It is therefore observed that the system will display an oscillatory behavior, tending to a (impulsively perturbed) limit cycle of period $T$ for an important portion of the parameter space, corresponding to impulsive and periodic perturbations with significant strength. It can also be observed that the well-known phenomenon of "exchange of stability" through simple bifurcation appears in our system, provided that a certain threshold condition is reached. 


\section{Preliminaries}

In this section, we shall introduce some notations and definitions and state some preliminary lemmas which will be useful for establishing our main results.

Let $R_{+}=[0, \infty), R_{+}^{2}=\left\{X \in R^{2}: X=\left(x_{1}, x_{2}\right), x_{1} \geq 0, x_{2} \geq 0\right\}$, and let $\mathbf{J} \subset R$. We introduce the following spaces of functions: $P C(\mathbf{J}, R) \doteq\left\{u: \mathbf{J} \rightarrow R: u\right.$ is continuous for $t \in \mathbf{J}, t \neq \tau_{k}$, continuous from the left for $t \in \mathbf{J}$, and has discontinuities of the first kind at the points $\tau k \in \mathbf{J}, \quad k \in \mathbf{N}\}$, and $P C^{1}(\mathbf{J}, R) \doteq\left\{u \in P C(\mathbf{J}, R): u\right.$ is continuously differentiable for $t \in \mathbf{J}, t \neq \tau_{k} ; u^{\prime}\left(\tau_{k}^{+}\right)$and $u^{\prime}\left(\tau_{k}^{-}\right)$ exist, $k \in \mathbf{N}$ \}. Denote by $f=\left(f_{1}, f_{2}\right)^{T}$ the map defined by the right hand side of the first two equations in the system (1).

Let $V: R_{+} \times R_{+}^{2} \rightarrow R_{+}$. Then $V$ is said to belong to class $V_{0}$ if

(i) $V$ is continuous on $((n-1) T,(n+\tilde{l}-1) T] \times R_{+}^{2} \quad$ and $\quad((n+\tilde{l}-1) T, n T] \times R_{+}^{2} \quad$ and $\quad$ for $\quad$ each $\quad X \in R_{+}^{2} \quad$ and $n \in \mathbf{N} \lim _{(t, Y) \rightarrow\left((n+\tilde{l}-1) T^{+}, X\right)} V(t, Y)=V\left((n+\tilde{l}-1) T^{+}, X\right)$ and $\lim _{(t, Y) \rightarrow\left(n T^{+}, X\right)} V(t, Y)=V\left(n T^{+}, X\right)$ exist and are finite. Here, $0<\tilde{l}<1$.

(ii) $V$ is locally Lipschitzian in the second variable.

Definition 3.1. Let $V \in V_{0}$. Then for $(t, X) \in((n-1) T$, $(n+\tilde{l}-1) T] \times R_{+}^{2}$ and $((n+\tilde{l}-1) T, n T] \times R_{+}^{2}(0<\tilde{l}<1)$, the upper right derivative of $V(t, x)$ with respect to the impulsive differential system (1) is defined as

$$
D^{+} V(t, X)=\lim _{h \rightarrow 0^{+}} \sup \frac{1}{h}[V(t+h, X+h f(t, X))-V(t, X)] .
$$

The solution of the system (1), denoted by $X(t)=(x(t), y(t)): R_{+} \rightarrow R_{+}^{2}$, is continuously differentiable on $((n-1) T,(n+\tilde{l}-$ 1)T] $\times R_{+}^{2}$ and $((n+\tilde{l}-1) T, n T] \times R_{+}^{2}$, with $0<\tilde{l}<1$. Obviously, the global existence and uniqueness of solutions of the system (1) is guaranteed by the smoothness properties of $f$ (see Lakshmikantham et al., 1989; Bainov and Simeonov, 1993 for details on fundamental properties of impulsive systems).

Lemma 3.2. The positive orthant $R_{+}^{2}$ is an invariant region for the system (1).

The proof of Lemma 3.2 is trivial and may be done by explicitly solving the initial value problem for the first two equations of the system (1) between pulses. Consequently, we omit it. The proofs of the following comparison and estimation lemmas are again obvious.

Lemma 3.3 (Bainov and Simeonov, 1993). Let $V: R_{+} \times R^{2} \rightarrow R_{+}$and $V \in V_{0}$. Assume that

$$
\begin{cases}D^{+} V(t, X) \leq g(t, V(t, X)), & t \neq(n+\tilde{l}-1) T, \quad t \neq n T, \\ V\left(t, X\left(t^{+}\right)\right) \leq \Psi_{n}^{(1)}(V(t, X(t))), & t=(n+\tilde{l}-1) T, \\ V\left(t, X\left(t^{+}\right)\right) \leq \Psi_{n}^{(2)}(V(t, X(t))), & t=n T,\end{cases}
$$

where $g: R_{+} \times R_{+}^{2} \rightarrow R_{+}^{2}$ is continuous on $((n-1) T,(n+\tilde{l}-1) T]$ and $((n+\tilde{l}-1) T, n T]$ and for each $v \in R_{+}^{2}, n \in N$,

$$
\lim _{(t, Y) \rightarrow\left((n+\tilde{l}-1) T^{+}, v\right)} g(t, Y)=g\left((n+\tilde{l}-1) T^{+}, v\right)
$$

and

$$
\lim _{(t, Y) \rightarrow\left(n T^{+}, v\right)} g(t, Y)=g\left(n T^{+}, v\right)
$$

exist and are finite, $\Psi_{n}^{(i)}(i=1,2): R_{+} \rightarrow R_{+}^{2}$ is quasi-monotone non-decreasing. Let $R\left(t, 0, U_{0}\right)$ be the maximal solution of the scalar impulsive differential equation

$$
\begin{cases}U^{\prime}(t)=g(t, U), & t \neq(n+\tilde{l}-1) T, \quad t \neq n T, \\ U\left(t^{+}\right)=\Psi_{n}^{(1)}(U(t)), & t=(n+\tilde{l}-1) T, \\ U\left(t^{+}\right)=\Psi_{n}^{(2)}(U(t)), & t=n T \\ U\left(0^{+}\right)=U_{0} & \end{cases}
$$

defined on $[0, \infty)$. Then $V\left(0^{+}, X_{0}\right) \leq U_{0}$ implies that $V(t, X(t)) \leq R(t), t \geq 0$, where $X(t)=X\left(t, 0, X_{0}\right)$ is any solution of $(1)$ defined on $[0, \infty)$. 
Proof. For $t \in[0, \tilde{l} T]$. By the classical comparison theorem for ordinary differential equations, we have $V(t, X(t)) \leq R(t)$. Since $\Psi_{1}^{(1)}$ is quasi-monotone non-decreasing and $V(\tilde{l} T, X(\tilde{l} T)) \leq R(\tilde{l} T)$, we obtain

$$
V\left(\tilde{l} T^{+}, X\left(\tilde{l} T^{+}\right)\right) \leq \Psi_{1}^{(1)}(V(\tilde{l} T, X(\tilde{l} T))) \leq \Psi_{1}^{(1)}(R(\tilde{l} T))=R\left(\tilde{l} T^{+}\right) .
$$

For $t \in \tilde{l} T, T]$, it follows, using again the classical comparison theorem for ordinary differential equations, that $V(t, X(t)) \leq R(t)$. Since $\Psi_{1}^{(2)}$ is quasi-monotone non-decreasing and $V(T, X(T)) \leq R(T)$, we get

$$
V\left(T^{+}, X\left(T^{+}\right)\right) \leq \Psi_{1}^{(2)}(V(T, X(T))) \leq \Psi_{1}^{(2)}(R(T))=R\left(T^{+}\right) .
$$

Thus, for $t \in[0, T]$, it follows that $V(t, X(t)) \leq R(t)$. Repeating this argument, we finally arrive at the desired result. This completes the proof.

Note that if we have some smoothness conditions on $g$ to guarantee the existence and uniqueness of solutions for (3), then $R(t)$ is exactly the unique solution of (3).

Lemma 3.4 (Bainov and Simeonov, 1993). Let the function $u \in P C^{1}\left(R_{+}, R\right)$ satisfy the inequalities

$$
\left\{\begin{array}{l}
u^{\prime}(t) \leq(\geq) \mathfrak{p}(t) u(t)+\mathrm{f}(t), \quad t \neq \tau_{k}, \quad t>0, \\
u\left(t^{+}\right) \leq(\geq) d_{k} u(t)+h_{k}, \quad t=\tau_{k}>0 \\
u\left(\tau_{0}^{+}\right)=u\left(0^{+}\right) \leq u_{0}
\end{array}\right.
$$

where $\mathfrak{p}, \mathrm{f} \in P C\left(R_{+}, R\right)$ and $d_{k} \geq 0, h_{k}$ and $u_{0}$ are constants.

Then, for $t>0$,

$$
u(t) \leq(\geq) u_{0} \prod_{0<\tau_{k}<t} d_{k} \exp \left(\int_{0}^{t} \mathfrak{p}(s) \mathrm{d} s\right)+\int_{0}^{t} \prod_{s \leq \tau_{k}<t} d_{k} \exp \left(\int_{s}^{t} \mathfrak{p}(\tau) \mathrm{d} \tau\right) \mathrm{f}(s) \mathrm{d} s+\sum_{0<\tau_{k}<t}\left(\prod_{\tau_{k}<\tau_{j}<t} d_{j} \exp \left(\int_{\tau_{k}}^{t} \mathfrak{p}(\tau) \mathrm{d} \tau\right)\right) h_{k} .
$$

Under these circumstances, it can be shown that the positive solutions of (1) are bounded, fact which is accomplished in the following Lemma.

Lemma 3.5. All solutions $(y(t), x(t))$ of (1) with initial data $(y(\cdot), x(\cdot)) \in R_{+}^{2}$ are bounded and defined on $R_{+}$.

\section{The stability of the prey (pest)-eradication periodic solution}

When the prey (pest) population $x$ is eradicated, it is easy to see that the equations in (1) decouple and we are led to consider the properties of the subsystem:

$$
\begin{cases}y^{\prime}(t)=-w y, & t \neq(n+\tilde{l}-1) T, \quad t \neq n T, \\ \triangle y(t)=-p_{2} y(t), & t=(n+\tilde{l}-1) T \\ \triangle y(t)=\mu, & t=n T \\ y\left(0^{+}\right)=y_{0}>0 . & \end{cases}
$$

It is seen that the system formed with the first three equations in (4) has a periodic solution to which all solutions of (4) starting with strictly positive $y_{0}$ tend as $t \rightarrow \infty$. This happens since the survival of the predator is assured by the periodic impulse $\mu$, which becomes the main driving force as far as the dynamics of the system (4) is concerned, and does not depend upon the survival or extinction of the prey.

Lemma 4.1. The system constructed with the first three equations of (4) has a positive periodic solution $y^{*}(t)$ with

$$
\int_{0}^{T} y^{*}(t) \mathrm{d} t=\frac{\mu}{w\left(1-\left(1-p_{2}\right) \mathrm{e}^{-w T}\right)}\left[\left(1-\mathrm{e}^{\tilde{I} T}\right)+\left(1-p_{2}\right)\left(\mathrm{e}^{-w \tilde{l} T}-\mathrm{e}^{-w T}\right)\right] .
$$

Moreover, for every solution $y(t)$ of the system (4), $\left|y(t)-y^{*}(t)\right| \rightarrow 0$ as $t \rightarrow \infty$.

Proof. The explicit form of the solution of (4) is

$$
y(t)= \begin{cases}\left(1-p_{2}\right)^{n-1}\left(y\left(0^{+}\right)-\frac{\mu}{1-\left(1-p_{2}\right) \exp (-w T)}\right) \exp (-w t)+y^{*}(t), & (n-1) T<t \leq(n+\tilde{l}-1) T, \\ \left(1-p_{2}\right)^{n}\left(y\left(0^{+}\right)-\frac{\mu}{1-\left(1-p_{2}\right) \exp (-w T)}\right) \exp (-w t)+y^{*}(t), & (n+\tilde{l}-1) T<t \leq n T,\end{cases}
$$


in which

$$
\begin{aligned}
& y^{*}(t)= \begin{cases}\frac{\mu \exp (-w(t-(n-1) T))}{1-\left(1-p_{2}\right) \exp (-w T)}, & (n-1) T<t \leq(n+\tilde{l}-1) T, \\
\frac{\mu\left(1-p_{2}\right) \exp (-w(t-(n-1) T))}{1-\left(1-p_{2}\right) \exp (-w T)}, & (n+\tilde{l}-1) T<t \leq n T,\end{cases} \\
& y^{*}\left(0^{+}\right)=y^{*}\left(n T^{+}\right)=\frac{\mu}{1-\left(1-p_{2}\right) \exp (-w T)}, \\
& y^{*}\left(\tilde{l} T^{+}\right)=\frac{\mu\left(1-p_{2}\right) \exp (-w \tilde{l} T)}{1-\left(1-p_{2}\right) \exp (-w T)} .
\end{aligned}
$$

The rest of the proof follows by an easy computation.

It is then easy to see that the system (1) has a so-called prey (pest)-eradication periodic solution $\left(y^{*}(t), 0\right)$. Next, we give conditions expressed in terms of an integral involving the periodic solution $y^{*}(t)$ which assure the local and global asymptotic stability of this prey (pest)-eradication periodic solution.

Theorem 4.2. The prey (pest)-eradication periodic solution $\left(y^{*}(t), 0\right)$ is locally stable $(L S)$ provided that

$$
\tilde{\mathcal{L}}\left(y^{*}(t)\right)=\int_{0}^{T} \frac{b y^{*}(t)}{A+k_{2} y^{*}(t)} \mathrm{d} t>T+\ln \left(1-p_{1}\right) .
$$

Further, $\left(y^{*}(t), 0\right)$ is globally asymptotically stable $(G A S)$ provided

$$
\mathcal{L}\left(y^{*}(t)\right)=\int_{0}^{T} \frac{b y^{*}(t)}{A+k_{1} K+k_{2} y^{*}(t)} \mathrm{d} t>T+\ln \left(1-p_{1}\right) .
$$

Proof. First, we prove the local stability result by using small amplitude perturbation methods. Let $(x(t), y(t))$ be a solution of (1) and let

$$
y(t)=v(t)+y^{*}(t), x(t)=u(t)
$$

where $u, v$ are understood to be small perturbations. The right-hand sides of the first two equations in (1) can be expanded using Taylor series. After neglecting higher-order terms, the linearized equations together with the corresponding impulsive perturbation conditions read as

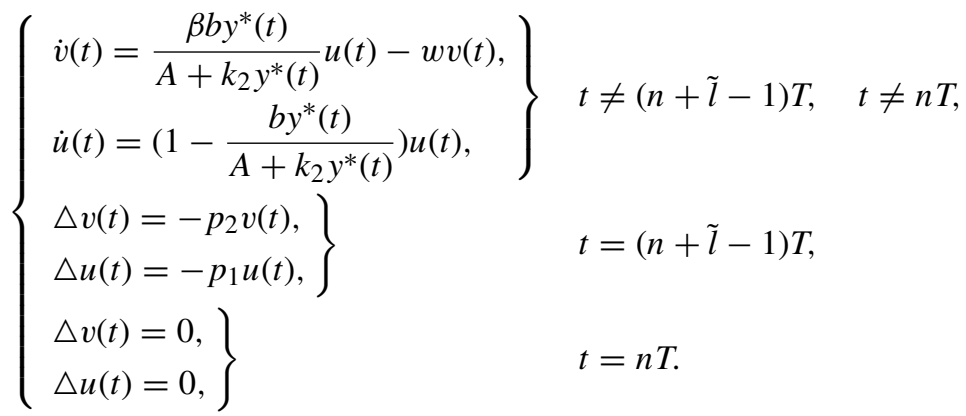

Let $\Phi(t)$ be the fundamental matrix of the subsystem formed with the first two equations in (7). Then $\Phi(t)$ must satisfy

$$
\frac{\mathrm{d} \Phi(t)}{\mathrm{d} t}=\left(\begin{array}{cc}
-w & \frac{\beta b y^{*}(t)}{A+k_{2} y^{*}(t)} \\
0 & 1-\frac{b y^{*}(t)}{A+k_{2} y^{*}(t)}
\end{array}\right) \Phi(t)
$$

and $\Phi(0)=E_{2}$ (unit $2 \times 2$ matrix). Hence the fundamental solution matrix $\Phi(t)$ is given by

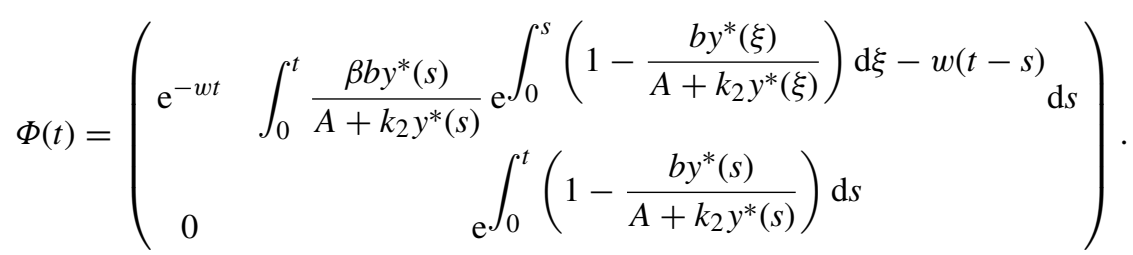


From the third and fourth equation of (7) one obtains that

$$
\left(\begin{array}{c}
v\left((n+\tilde{l}-1) T^{+}\right) \\
u\left((n+\tilde{l}-1) T^{+}\right)
\end{array}\right)=\left(\begin{array}{cc}
1-p_{2} & 0 \\
0 & 1-p_{1}
\end{array}\right)\left(\begin{array}{c}
v((n+\tilde{l}-1) T) \\
u((n+\tilde{l}-1) T)
\end{array}\right),
$$

while from the fifth and sixth equation of (7) one obtains that

$$
\left(\begin{array}{l}
v\left(n T^{+}\right) \\
u\left(n T^{+}\right)
\end{array}\right)=\left(\begin{array}{ll}
1 & 0 \\
0 & 1
\end{array}\right)\left(\begin{array}{c}
v(n T) \\
u(n T)
\end{array}\right) .
$$

Hence, if each eigenvalue of the transition matrix

$$
M^{*}=\left(\begin{array}{cc}
1-p_{2} & 0 \\
0 & 1-p_{1}
\end{array}\right)\left(\begin{array}{ll}
1 & 0 \\
0 & 1
\end{array}\right) \Phi(T)
$$

has absolute value less than one, then the periodic solution $\left(y^{*}(t), 0\right)$ is locally stable. Since the eigenvalues of $M^{*}$ are

$$
\begin{aligned}
& \lambda_{1}=\left(1-p_{2}\right) \exp (-w T)<1, \quad \lambda_{2}=\left(1-p_{1}\right) \exp \left(\int_{0}^{T}\left(1-\frac{b y^{*}(s)}{A+k_{2} y^{*}(s)}\right) \mathrm{d} s\right) . \\
& \lambda_{2}<1 \Leftarrow \int_{0}^{T}\left(\frac{b y^{*}(s)}{A+k_{2} y^{*}(s)}\right) \mathrm{d} s\left(>\int_{0}^{T}\left(\frac{b y^{*}(s)}{A+k_{1} K+k_{2} y^{*}(s)}\right) \mathrm{d} s\right)>T+\ln \left(1-p_{1}\right)
\end{aligned}
$$

Hence $\lambda_{2}<1$ if (5) holds. According to the Floquet theory of impulsive differential equation, the prey-eradication solution $\left(y^{*}(t), 0\right)$ is then locally stable.

In the following we shall prove its global attractivity provided that condition (6) is satisfied. In regard to this, choose an $\epsilon>0$ such that

$$
\zeta \doteq\left(1-p_{1}\right) \exp \left(\int_{0}^{T}\left(1-\frac{b\left(y^{*}(t)-\epsilon\right)}{A+k_{1}(K+\epsilon)+k_{2}\left(y^{*}(t)-\epsilon\right)}\right) \mathrm{d} s\right)<1 .
$$

Noting that $y^{\prime}(t) \geq-w y(t)$, from Lemma 3.4 and the comparison theorem for impulsive differential equations (Lakshmikantham et al., 1989; Bainov and Simeonov, 1993), we get

$$
y(t)>y^{*}(t)-\epsilon
$$

for all $t$ large enough. For simplicity, we may assume that (9) holds for all $t \geq 0$. It is also easy to see that $\lim _{\sup } \operatorname{su}_{t \rightarrow \infty} x(t) \leq K$ and consequently $x(t)<K+\epsilon$ for all $t$ large enough. Again, for simplicity, we may assume that $x(t)<K+\epsilon$ for all $t \geq 0$. From (1) and (9), we deduce

$$
\begin{cases}x^{\prime}(t) \leq x(t)\left(1-\frac{b\left(y^{*}(t)-\epsilon\right)}{A+k_{1}(K+\epsilon)+k_{2}\left(y^{*}(t)-\epsilon\right)}\right), & t \neq(n+\tilde{l}-1) T, \\ x\left(t^{+}\right)=\left(1-p_{1}\right) x(t), & t=(n+\tilde{l}-1) T .\end{cases}
$$

Integrating $(10)$ on $((n+\tilde{l}-1) T,(n+\tilde{l}) T]$, one obtains

$$
x((n+\tilde{l}) T) \leq x((n+\tilde{l}-1) T)\left(1-p_{1}\right) \exp \left(\int_{(n+\tilde{l}-1) T}^{(n+\tilde{l}) T}\left(1-\frac{b\left(y^{*}(t)-\epsilon\right)}{A+k_{1}(K+\varepsilon)+k_{2}\left(y^{*}(t)-\epsilon\right)}\right) \mathrm{d} t\right)=x((n+\tilde{l}-1) T) \zeta .
$$

Thus $x((n+\tilde{l}) T) \leq x(\tilde{l} T) \zeta^{n}$ and consequently $x((n+\tilde{l}) T) \rightarrow 0$ as $n \rightarrow \infty$. Hence, $x(t) \rightarrow 0$ as $t \rightarrow \infty$ since $0<x(t) \leq x((n+$ $\tilde{l}-1) T)\left(1-p_{1}\right) \exp (T)$ for $t \in((n+\tilde{l}-1) T,(n+\tilde{l}) T]$.

Next, we prove that $y(t) \rightarrow y^{*}(t)$ as $t \rightarrow \infty$. For $0<\epsilon^{\prime}<(w A / \beta b)$, there exists a $\tilde{T}>0$ such that $0<x(t)<\epsilon^{\prime}$ for $t \geq \tilde{T}$. Without loss of generality, we may assume that $0<x(t)<\epsilon^{\prime}$ for $t \geq 0$. Then, from (1), we have

$$
-w y(t) \leq y^{\prime}(t)<\left(-w+\frac{\beta b \epsilon^{\prime}}{A}\right) y(t) .
$$


From Lemmas 3.4 and 4.1, we obtain $y_{1}(t) \leq y(t) \leq y_{2}(t)$ and $y_{1}(t) \rightarrow y^{*}(t), y_{2}(t) \rightarrow y_{2}^{*}(t)$ as $t \rightarrow \infty$, where $y_{1}(t)$ and $y_{2}(t)$ are the solutions of

$$
\begin{cases}y_{1}^{\prime}(t)=-w y_{1}(t), & t \neq(n+\tilde{l}-1) T, \quad t \neq n T, \\ \triangle y_{1}(t)=-p_{2} y_{1}(t), & t=(n+\tilde{l}-1) T, \\ \triangle y_{1}(t)=\mu, & t=n T, \\ y_{1}\left(0^{+}\right)=y_{0} & \end{cases}
$$

and

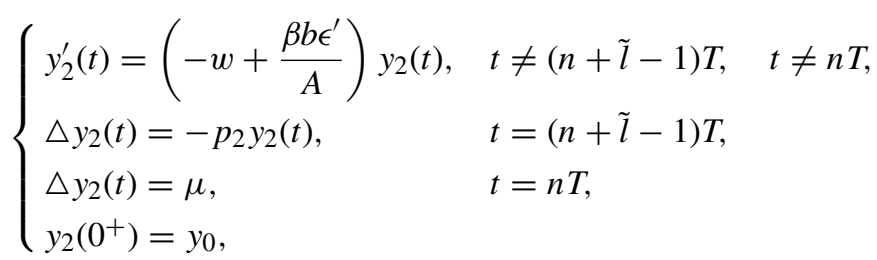

respectively, where

$$
y_{2}^{*}(t)=\left\{\begin{array}{l}
\frac{\mu \exp \left(\left(-w+\left(\beta b \epsilon^{\prime} / A\right)\right)(t-(n-1) T)\right)}{1-\left(1-p_{2}\right) \exp \left(\left(-w+\left(\beta b \epsilon^{\prime} / A\right)\right) T\right)}, \quad(n-1) T<t \leq(n+\tilde{l}-1) T, \\
\frac{\mu\left(1-p_{2}\right) \exp \left(\left(-w+\left(\beta b \epsilon^{\prime} / A\right)\right)(t-(n-1) T)\right)}{1-\left(1-p_{2}\right) \exp \left(\left(-w+\left(\beta b \epsilon^{\prime} / A\right)\right) T\right)}, \quad(n+\tilde{l}-1) T<t \leq n T .
\end{array}\right.
$$

Therefore,

$$
y^{*}(t)-\varepsilon_{1}<y(t)<y_{2}^{*}(t)+\varepsilon_{1}, \quad\left(\varepsilon_{1}>0\right)
$$

for $t$ large enough. Let $\epsilon^{\prime} \rightarrow 0$, we get $y_{2}^{*}(t) \rightarrow y^{*}(t)$. Hence $y(t) \rightarrow y^{*}(t)$ as $t \rightarrow \infty$. This completes the proof.

Remark 4.3. One easily sees from Theorem 4.2 that if $T+\ln \left(1-p_{1}\right) \leq 0$, then $\left(y^{*}(t), 0\right)$ is globally asymptotically stable. Also, if $\tilde{\mathcal{L}}\left(y^{*}(t)\right)>\left(T+\ln \left(1-p_{1}\right)\right)$, then $\left(y^{*}(t), 0\right)$ is unstable.

Remark 4.4. Here the LS of the pest-eradication periodic solution of the system (1) is independent of the carrying capacity $K$ of the pest population, while its GAS depends on the effect of the carrying capacity $\mathrm{K}$.

We rewrite the prey equation in the system (1) and obtain the following equation

$$
x^{\prime}(t)=x(t)\left(\left[1-\frac{b y(t)}{A+k_{1} x(t)+k_{2} y(t)}\right]-\frac{x(t)}{K}\right) .
$$

It is easy to seen that the integral terms

$$
-\int_{0}^{T} \frac{b y^{*}(t)}{A+k_{2} y^{*}(t)} \mathrm{d} t
$$

and

$$
-\int_{0}^{T} \frac{b y^{*}(t)}{A+k_{1} K+k_{2} y^{*}(t)} \mathrm{d} t
$$

approximate the (per pest unit biomass) maximal and, respectively, minimal additional loss of pests in a period $T$ due to predator consumption, while since the production of newborn pests in the absence of predation is given by $x(\underbrace{r}_{=1}-(x / K)), r T=T$ approximates the (per pest unit biomass) intrinsic growth of pests in a period. Also $\ln \left(1-p_{1}\right)$ is a correction term which accounts for the loss of pest biomass (per pest unit biomass) due to pesticide spraying. Then if $T+\ln \left(1-p_{1}\right) \leq 0$, that is, the total growth $T$ does not exceed the loss $\ln \left(1-p_{1}\right)$, there is a total net loss of pest biomass when $x$ approaches 0 and so the prey (pest) biomass $x(t)$ tends to 0 as $t \rightarrow \infty$. If $\tilde{\mathcal{L}}\left(y^{*}(t)\right)>\ln \left(1-p_{1}\right)+T$, then the loss of pest biomass in a period $T$ near $\left(y^{*}(t), 0\right)$ exceeds the total pest growth in the same amount of time, so the prey (pest)-eradication periodic solution is locally stable. A similar interpretation can be performed with respect to the condition $\mathcal{L}\left(y^{*}(t)\right)>\ln \left(1-p_{1}\right)+T$.

\section{A sufficient condition for permanence}

Uniform persistence (or permanence) is an important property of dynamical systems and of the ecosystems. It is actually a concept which is important in itself, addressing the long-term survival of some or all components of a system. In this section we shall point out a sufficient condition for the permanence of system (1). 
Definition 5.1. The system (1) is said to be permanent if there are constants $m, M>0$ (independent of the initial data $x\left(0^{+}\right)>0$ and $y\left(0^{+}\right)>0$ ) and a finite time $T_{0}$ (which may depend on the initial data) such that $m \leq x(t), y(t) \leq M$ for all solutions $(y(t), x(t)$ ) and $t \geq T_{0}$.

Theorem 5.2. The system (1) is permanent provided that

$$
\tilde{\mathcal{L}}\left(y^{*}(t)\right)=\int_{0}^{T} \frac{b y^{*}(t)}{A+k_{2} y^{*}(t)} \mathrm{d} t<T+\ln \left(1-p_{1}\right),
$$

holds.

Proof. Suppose that $(y(t), x(t))$ is a solution of (1) with $x(0)>0, y(0)>0$. From Lemma 3.5, there exists a constant $M>0$ such that $x(t) \leq M, y(t) \leq M$ for $t \geq 0$.

From (9), we note that $y(t)>y^{*}(t)-\epsilon$ for all $t$ large enough. Consequently, $y(t) \geq\left(\left(\mu\left(1-p_{2}\right) \exp (-w T)\right) /(1-\exp (-w T))\right)-$ $\varepsilon \doteq m_{2}$ for $t$ large enough. We then only need to find $m_{1}>0$, such that $x(t) \geq m_{1}$ for large enough. We will do it in the following two steps for convenience.

Step I. Let $0<m_{3}<(A w / \beta b)$ be small enough and suppose that $x(t) \leq m_{3}$ for all $t \geq 0$. We then obtain $y(t) \leq y_{3}(t)$ and $y_{3}(t) \rightarrow y_{3}^{*}(t)$ as $t \rightarrow \infty$, where $y_{3}(t)$ is the solution of

$$
\left\{\begin{array}{lll}
y_{3}^{\prime}(t)=\left(-w+\frac{\beta b m_{3}}{A+k_{1} m_{3}+k_{2} m_{2}}\right) y_{3}(t), & t \neq(n+\tilde{l}-1) T, \quad t \neq n T \\
\triangle y_{3}(t)=-p_{2} y_{3}(t), & t=(n+\tilde{l}-1) T \\
\triangle y_{3}(t)=\mu, & t=n T \\
y_{3}\left(0^{+}\right)=y_{0} &
\end{array}\right.
$$

and

$$
y_{3}^{*}(t)=\left\{\begin{array}{l}
\frac{\mu \exp \left(\left(-w+\left(\beta b m_{3} /\left(A+k_{1} m_{3}+k_{2} m_{2}\right)\right)\right)(t-(n-1) T)\right)}{1-\left(1-p_{2}\right) \exp \left(\left(-w+\left(\beta b m_{3} /\left(A+k_{1} m_{3}+k_{2} m_{2}\right)\right)\right) T\right)}, \quad(n-1) T<t \leq(n+\tilde{l}-1) T, \\
\frac{\mu\left(1-p_{2}\right) \exp \left(\left(-w+\left(\beta b m_{3} /\left(A+k_{1} m_{3}+k_{2} m_{2}\right)\right)\right)(t-(n-1) T)\right)}{1-\left(1-p_{2}\right) \exp \left(\left(-w+\left(\beta b m_{3} /\left(A+k_{1} m_{3}+k_{2} m_{2}\right)\right)\right) T\right)},(n+\tilde{l}-1) T<t \leq n T .
\end{array}\right.
$$

Consequently, there exists $\hat{T}>0$ such that

$$
y(t) \leq y_{3}(t)<y_{3}^{*}(t)+\varepsilon \text { for } t \geq \hat{T},
$$

where $\varepsilon$ is small enough, so that

$$
\left(1-p_{1}\right) \exp \left(\int_{0}^{T}\left(1-\frac{b\left(y_{3}^{*}(t)+\varepsilon\right)}{A+k_{2}\left(y_{3}^{*}(t)+\varepsilon\right)}\right) \mathrm{d} t\right)>1 .
$$

On the other hand, from (1), we have

$$
\begin{cases}x^{\prime}(t) \geq\left(1-\frac{b\left(y_{3}^{*}(t)+\varepsilon\right)}{A+k_{2}\left(y_{3}^{*}(t)+\varepsilon\right)}\right) x(t), & t \neq(n+\tilde{l}-1) T, \\ \triangle x(t)=-p_{1} x(t), & t=(n+\tilde{l}-1) T,\end{cases}
$$

for $t \geq \hat{T}$. Let $N \in \mathbf{N}^{*}$ such that $(N+\tilde{l}-1) T \geq \hat{T}$. Integrating (14) on $((n+\tilde{l}-1) T,(n+\tilde{l}) T], n \geq N$, we get

$$
x((n+\tilde{l}) T) \geq x((n+\tilde{l}-1) T)\left(1-p_{1}\right) \exp \left(\int_{(n+\tilde{l}-1) T}^{(n+\tilde{l}) T}\left(1-\frac{b\left(y_{3}^{*}(t)+\varepsilon\right)}{A+k_{2}\left(y_{3}^{*}(t)+\varepsilon\right)}\right) \mathrm{d} t\right)=x((n+\tilde{l}-1) T) \eta .
$$

One then obtains that $x((N+n+\tilde{l}-1) T) \geq x((N+\tilde{l}) T) \eta^{n} \rightarrow \infty$ as $n \rightarrow \infty$, which contradicts the boundedness of $x(t)$. Thus, there exist a $t_{1}>0$ such that $x\left(t_{1}\right) \geq m_{3}$. The next step of proof is similar to the one in Theorem 4.3 in Zhang et al. (2007a). To avoid redundancy, we omit it. This completes the proof.

Remark 5.3. From Theorems 4.2 and 5.2, one sees that if $\tilde{\mathcal{L}}\left(y^{*}(t)\right)-\ln \left(1-p_{1}\right)>T$, then the prey (pest)-eradication periodic solution $\left(y^{*}, 0\right)$ is locally asymptotically stable, while if $\tilde{\mathcal{L}}\left(y^{*}(t)\right)-\ln \left(1-p_{1}\right)<T$, then the prey (pest)-eradication periodic solution $\left(y^{*}, 0\right)$ loses its stability and the system becomes permanent.

Remark 5.4. The biological interpretation of Theorem 5.2 is that once the total growth of pest biomass (per pest unit biomass) in a period $T$ exceeds the total maximal loss of pest biomass (per pest unit biomass) in a period $\tilde{\mathcal{L}}\left(y^{*}(t)\right)-\ln \left(1-p_{1}\right)$, then the pest population and its natural enemy will coexist forever. 


\section{The bifurcation of a nontrivial periodic solution}

In the following, we shall study the loss of stability phenomenon mentioned in Remark 5.3 and prove that it is due to the onset of nontrivial periodic solutions obtained via a supercritical bifurcation in the limiting case, that is,

$$
\tilde{\mathcal{L}}\left(y^{*}(t)\right)=T+\ln \left(1-p_{1}\right),
$$

by means of bifurcation theory methods. Here, by "nontrivial periodic solution" we mean a periodic solution which is different from $\left(y^{*}, 0\right)$. First, we shall denote by $\Phi\left(t ; X_{0}\right)$ the solution of the pulses-free system corresponding to system (1) with the initial data $\left(x_{0}^{1}, x_{0}^{2}\right)$; also $\Phi=\left(\Phi_{1}, \Phi_{2}\right)$. We also define two maps $I_{1}, I_{2}: R^{2} \rightarrow R^{2}$ by

$$
I_{1}\left(x_{1}, x_{2}\right)=\left(\left(1-p_{2}\right) x_{1},\left(1-p_{1}\right) x_{2}\right), I_{2}\left(x_{1}, x_{2}\right)=\left(x_{1}+\mu, x_{2}\right) .
$$

and the map $F: R^{2} \rightarrow R^{2}$ by

$$
F\left(x_{1}, x_{2}\right)=\left(\frac{\beta b x_{1} x_{2}}{A+k_{1} x_{2}+k_{2} x_{1}}-w x_{1}, x_{2}\left(1-x_{2} / K\right)-\frac{b x_{1} x_{2}}{A+k_{1} x_{2}+k_{2} x_{1}}\right) .
$$

In the following, we shall reduce the problem of finding a periodic solution of (1) to a certain fixed point problem. To this purpose, define $\Psi:[0, \infty) \times R^{2} \rightarrow R^{2}$ by

$$
\Psi\left(t, X_{0}\right)=I_{2}\left((1-\tilde{l}) T ; I_{1}\left(\tilde{l} T, X_{0}\right)\right) ;
$$

also

$$
\Psi\left(t, X_{0}\right)=\left(\Psi_{1}\left(t, X_{0}\right), \Psi_{2}\left(t, X_{0}\right)\right) .
$$

Then $X$ is a $T$-periodic solution of the system (1) if and only if its initial data $X(0)=X_{0}$ is a fixed point for the operator $\Psi$. Hence, to study the existence of nontrivial periodic solutions for (1), we need to study the existence of nontrivial fixed points for the operator $\Psi$.

Next, we note that

$$
D_{X} \Psi(T, X)=D_{X} \Phi\left((1-\tilde{l}) T ; I_{1}(\Phi(\tilde{l} T, X))\right)\left(\begin{array}{cc}
1-p_{2} & 0 \\
0 & 1-p_{1}
\end{array}\right) D_{X} \Phi(\tilde{l} T ; X) .
$$

Let $X_{0}=\left(x_{0}, 0\right)$ be the initial condition corresponding to the trivial periodic solution $\left(y^{*}(t), 0\right)$, where $x_{0}=y^{*}(0)$. By employing the techniques developed in Lakmeche and Arino (2000), we now consider the bifurcation of nontrivial periodic solutions near $\left(y^{*}(t), 0\right)$. To this purpose, we need to carry on certain computations. One easily obtains that

$$
D_{X} \Psi\left(T, X_{0}\right)=\left(\begin{array}{cc}
d_{11} & d_{12} \\
0 & d_{22}
\end{array}\right)
$$

in which

$$
\begin{aligned}
& d_{11}=\left(1-p_{2}\right) \mathrm{e}^{-w T} ; \\
& d_{12}=\mathrm{e}^{-w T}\left(\left(1-p_{1}\right) \int_{\tilde{l} T}^{T} \beta \mathcal{P}\left(y^{*}(s)\right) \mathrm{e}^{(w+1)-\int_{0}^{s} \mathcal{P}\left(y^{*}(\xi)\right) \mathrm{d} \xi} \mathrm{d} s+\left(1-p_{2}\right) \int_{0}^{\tilde{l} T} \beta \mathcal{P}\left(y^{*}(s)\right) \mathrm{e}^{(w+1)-\int_{0}^{s} \mathcal{P}\left(y^{*}(\xi)\right) \mathrm{d} \xi} \mathrm{d} s\right) ; \\
& d_{22}=\left(1-p_{1}\right) \mathrm{e}^{T-\int_{0}^{T} \mathcal{P}\left(y^{*}(s)\right) \mathrm{d} s},
\end{aligned}
$$

in which

$$
\mathcal{P}\left(y^{*}(s)\right)=\frac{b y^{*}(s)}{A+k_{2} y^{*}(s)} .
$$

Let us denote

$$
\tau=T+\bar{\tau}, \quad X=X_{0}+\bar{X} .
$$
is,

To find a nontrivial periodic solution of period $\tau$ with initial data $X$, we need to solve the fixed point problem $X=\Psi(\tau, X)$, that

$$
X_{0}+\bar{X}=\Psi\left(T+\bar{\tau}, X_{0}+\bar{X}\right) .
$$


Let

$$
N(\bar{\tau}, \bar{X})=X_{0}+\bar{X}-\Psi\left(T+\bar{\tau}, X_{0}+\bar{X}\right)
$$

and

$$
N(\bar{\tau}, \bar{X})=\left(N_{1}(\bar{\tau}, \bar{X}), N_{2}(\bar{\tau}, \bar{X})\right) .
$$

We are then led to solve the equation $N(\bar{\tau}, \bar{X})=0$. One notes that

$$
D_{X} N(0,(0,0))=E_{2}-D_{X} \Psi\left(T, X_{0}\right)=\left(\begin{array}{cc}
1-d_{11} & -d_{12} \\
0 & 1-d_{22}
\end{array}\right)=\left(\begin{array}{cc}
a_{0}^{\prime} & b_{0}^{\prime} \\
0 & d_{0}^{\prime}
\end{array}\right) .
$$

A necessary condition for the bifurcation of the nontrivial periodic solutions near the trivial periodic solution $\left(y^{*}(t), 0\right)$ is

$$
\operatorname{det}\left[D_{X} N(0,(0,0))\right]=0 .
$$

Since $a_{0}^{\prime} \neq 0$, it follows that $d_{0}^{\prime}=0$, that is,

$$
\left(1-p_{1}\right) \mathrm{e}^{T-\int_{0}^{T} \mathcal{P}\left(y^{*}(s)\right) \mathrm{d} s}=1 \Leftrightarrow \tilde{\mathcal{L}}\left(y^{*}(t)\right)=T+\ln \left(1-p_{1}\right) .
$$

It is seen that

$$
\operatorname{dim}\left(\operatorname{Ker}\left[D_{X}(0,(0,0))\right]\right)=1,
$$

and a basis in $\operatorname{Ker}\left[D_{X}(0,(0,0))\right]$ is $\left(-b_{0}^{\prime} / a_{0}^{\prime}, 1\right)$. Then the equation $N(\bar{\tau}, \bar{X})=0$ is equivalent to

$$
\left\{\begin{array}{l}
N_{1}\left(\bar{\tau}, \alpha Y_{0}+z E_{0}\right)=0 \\
N_{2}\left(\bar{\tau}, \alpha Y_{0}+z E_{0}\right)=0
\end{array}\right.
$$

where

$$
E_{0}=(1,0), Y_{0}=\left(-b_{0}^{\prime} / a_{0}^{\prime}, 1\right)
$$

and $\bar{X}=\alpha Y_{0}+z E_{0}=\left(\alpha\left(-b_{0}^{\prime} / a_{0}^{\prime}\right)+z, \alpha\right)$ represents the direct sum decomposition of $X$ using the projections onto $\operatorname{Ker}\left[D_{X} N(0,(0,0))\right]$ and $\operatorname{Im}\left[D_{X} N(0,(0,0))\right]$ (Chow and Hale, 1982).

Let

$$
\begin{aligned}
& f_{1}(\bar{\tau}, \alpha, z)=N_{1}\left(\bar{\tau}, \alpha Y_{0}+z E_{0}\right), \\
& f_{2}(\bar{\tau}, \alpha, z)=N_{2}\left(\bar{\tau}, \alpha Y_{0}+z E_{0}\right) .
\end{aligned}
$$

We now need to solve the following system

$$
\left\{\begin{array}{l}
f_{1}(\bar{\tau}, \alpha, z)=0 \\
f_{2}(\bar{\tau}, \alpha, z)=0
\end{array}\right.
$$

Since

$$
\frac{\partial f_{1}}{\partial z}(0,0,0)=\frac{\partial N_{1}}{\partial x_{1}}(0,(0,0))=a_{0}^{\prime} \neq 0
$$

by applying the implicit function theorem, one may locally solve the equation $f_{1}(\bar{\tau}, \alpha, z)=0$ near $(0,0,0)$ with respect to $z$ as a function of $\bar{\tau}$ and $\alpha$ and find $z=z(\bar{\tau}, \alpha)$ such that $z(0,0)=0$ and

$$
f_{1}(\bar{\tau}, \alpha, z(\bar{\tau}, \alpha))=N_{1}\left(\bar{\tau}, \alpha Y_{0}+z(\bar{\tau}, \alpha) E_{0}\right)=0 .
$$

One may then deduce that

$$
\frac{\partial N_{1}}{\partial x_{1}}\left(\frac{\partial x_{1}}{\partial \alpha}+\frac{\partial x_{1}}{\partial z} \frac{\partial z}{\partial \alpha}(0,0)\right)+\frac{\partial N_{2}}{\partial x_{2}} \frac{\partial x_{2}}{\partial \alpha}=0 .
$$

It follows from (17) that

$$
\frac{\partial z}{\partial \alpha}(0,0)=0 \text {. }
$$


In view of (16), we get that

$$
\begin{aligned}
\frac{\partial z}{\partial \bar{\tau}}(0,0)= & \frac{\partial I_{2}}{\partial x_{1}} \frac{\partial \Phi_{1}}{\partial \bar{\tau}}\left((1-\tilde{l}) T ; I_{1}\left(\Phi\left(\tilde{l} T ; X_{0}\right)\right)\right)(1-\tilde{l}) \\
& +\frac{\partial I_{2}}{\partial x_{1}} \frac{\partial \Phi_{1}}{\partial x_{1}}\left((1-\tilde{l}) T ; I_{1}\left(\Phi\left(\tilde{l} T ; X_{0}\right)\right)\right)\left(1-p_{2}\right)\left(\frac{\partial \Phi_{1}}{\partial \bar{\tau}}\left(\tilde{l} T ; X_{0}\right) \cdot \tilde{l}+\frac{\partial \Phi_{1}}{\partial x_{1}}\left(\tilde{l} T ; X_{0}\right) \frac{\partial z}{\partial \bar{\tau}}(0,0)\right) \\
& \left.+\frac{\partial I_{2}}{\partial x_{1}} \frac{\partial \Phi_{1}}{\partial x_{2}}\left((1-\tilde{l}) T ; I_{1}\left(\Phi\left(\tilde{l} T ; X_{0}\right)\right)\right)\left(1-p_{1}\right)\left(\frac{\partial \Phi_{2}}{\partial \bar{\tau}}\left(\tilde{l} T ; X_{0}\right) \cdot \tilde{l}+\frac{\partial \Phi_{2}}{\partial x_{1}} \tilde{l} T ; X_{0}\right) \frac{\partial z}{\partial \bar{\tau}}(0,0)\right) .
\end{aligned}
$$

Then

$$
\begin{aligned}
& \frac{\partial z}{\partial \bar{\tau}}(0,0)\left(1-\frac{\partial \Phi_{1}}{\partial x_{1}}\left((1-\tilde{l}) T ; I_{1}\left(\Phi\left(\tilde{l} T ; X_{0}\right)\right)\right)\left(1-p_{2}\right) \frac{\partial \Phi_{1}}{\partial x_{1}}\left(\tilde{l} T ; X_{0}\right)\right) \\
& \quad=\frac{\partial \Phi_{1}}{\partial \bar{\tau}}\left((1-\tilde{l}) T ; I_{1}\left(\Phi\left(\tilde{l} T ; X_{0}\right)\right)\right)(1-\tilde{l})+\frac{\partial \Phi_{1}}{\partial x_{1}}\left((1-\tilde{l}) T ; I_{1}\left(\Phi\left(\tilde{l} T ; X_{0}\right)\right)\right)\left(1-p_{2}\right) \frac{\partial \Phi_{1}}{\partial \bar{\tau}}\left(\tilde{l} T ; X_{0}\right) \cdot \tilde{l} .
\end{aligned}
$$

It is easily seen that

$$
\frac{\partial z}{\partial \bar{\tau}}(0,0)=\frac{1}{a_{0}^{\prime}}\left[-w y^{*}(T)(1-\tilde{l})+\left(1-p_{2}\right) \mathrm{e}^{-w(1-\tilde{l}) T}\left(-w y^{*}(\tilde{l} T)\right) \cdot \tilde{l}\right]=-\frac{w}{a_{0}^{\prime}} y^{*}(T) .
$$

It now remains to study the solvability of the equation

$$
f_{2}(\bar{\tau}, \alpha, z(\bar{\tau}, \alpha))=N_{2}\left(\bar{\tau}, \alpha Y_{0}+z(\bar{\tau}, \alpha) E_{0}\right)=0 .
$$

Eq. (22) is called the determining equation and the number of its solutions equals the number of periodic solutions of (1)(Chow and Hale, 1982). Next, we proceed to solving (22) by using Taylor expansions. We denote

$$
f(\bar{\tau}, \alpha)=f_{2}(\bar{\tau}, \alpha, z(\bar{\tau}, \alpha)) .
$$

First, we observe that

$$
f(0,0)=N_{2}(0,(0,0))=0 .
$$

Second, we focus on the first order partial derivatives of $f$ at $(0,0)$. From Appendix A.1 it is easy to see that

$$
\frac{\partial f}{\partial \alpha}(0,0)=1-\frac{\partial \Phi_{2}}{\partial x_{2}}\left((1-\tilde{l}) T ; I_{1}\left(\Phi\left(\tilde{l} T ; X_{0}\right)\right)\right)\left(1-p_{1}\right) \frac{\partial \Phi_{2}}{\partial x_{2}}\left(\tilde{l} T ; X_{0}\right)=d_{0}^{\prime}=0 .
$$

It consequently follows that

$$
\frac{\partial f}{\partial \bar{\tau}}(0,0)=0 .
$$

Third, we compute the second-order partial derivatives $\left(\partial^{2} f / \partial \alpha^{2}\right),\left(\partial^{2} f / \partial \bar{\tau}^{2}\right)$ and $\left(\partial^{2} f / \partial \alpha \partial \bar{\tau}\right)$.

According to the computations in Appendix A.2, we get that

$$
\begin{aligned}
& \frac{\partial^{2} f}{\partial \bar{\tau}^{2}}(0,0)=-\frac{\partial^{2} \Phi_{2}}{\partial \bar{\tau}^{2}}\left((1-\tilde{l}) T ; I_{1}\left(\Phi\left(\tilde{l} T ; X_{0}\right)\right)\right)(1-\tilde{l})^{2}=0, \\
& \frac{\partial^{2} f}{\partial \alpha^{2}}(0,0)>0,
\end{aligned}
$$

and

$$
\frac{\partial^{2} f}{\partial \bar{\tau} \partial \alpha}(0,0)<0
$$

By constructing the second-order Taylor expansion of $f$ near $(0,0)$, one obtains from (24)-(28) that

$$
f(\bar{\tau}, \alpha)=\underbrace{\frac{\partial^{2} f}{\partial \bar{\tau} \partial \alpha}(0,0)}_{<0} \alpha \bar{\tau}+\frac{1}{2} \underbrace{\frac{\partial^{2} f}{\partial \alpha^{2}}(0,0)}_{>0} \alpha^{2}+o(\bar{\tau}, \alpha)\left(\bar{\tau}^{2}+\alpha^{2}\right) .
$$


Table 1

Threshold values for the stability and permanence of the system, with regard to $p_{1}$ and $\mu$

$p_{2}=0.01, w=0.5, b=1.5, T=2, k_{1}=8$,

GAS of the pest-free periodic solution $\left(y^{*}(t), 0\right)$

Permanence

$k_{2}=0.1, A=2, K=1.6, \tilde{l}=0.5$

$\mu=1$

$p_{1}>0.8347011118$

$p_{1}<0.4511883639$

$p_{1}=1-\mathrm{e}^{-1.9}$
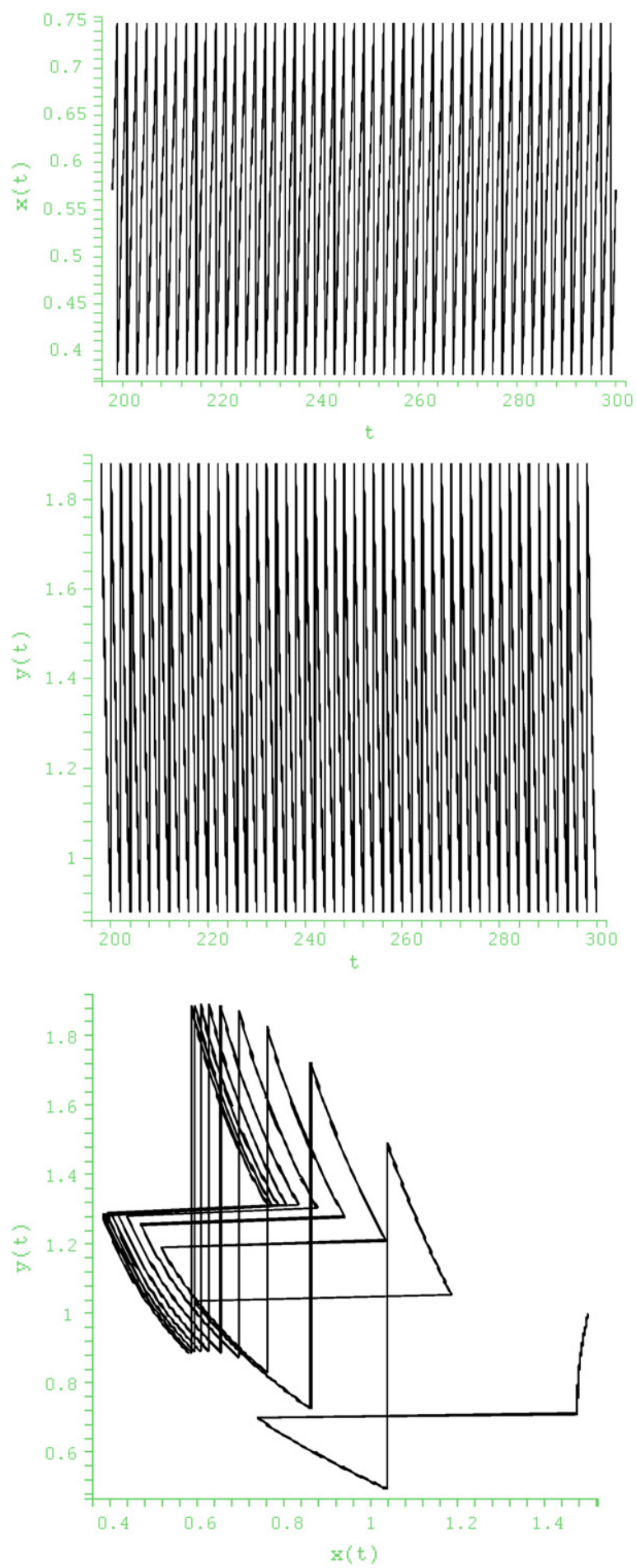

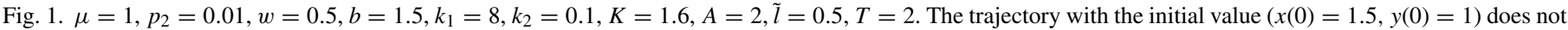
approach the periodic trivial orbit of period $T$ at $p_{1}=1-\exp (-0.7)$. 

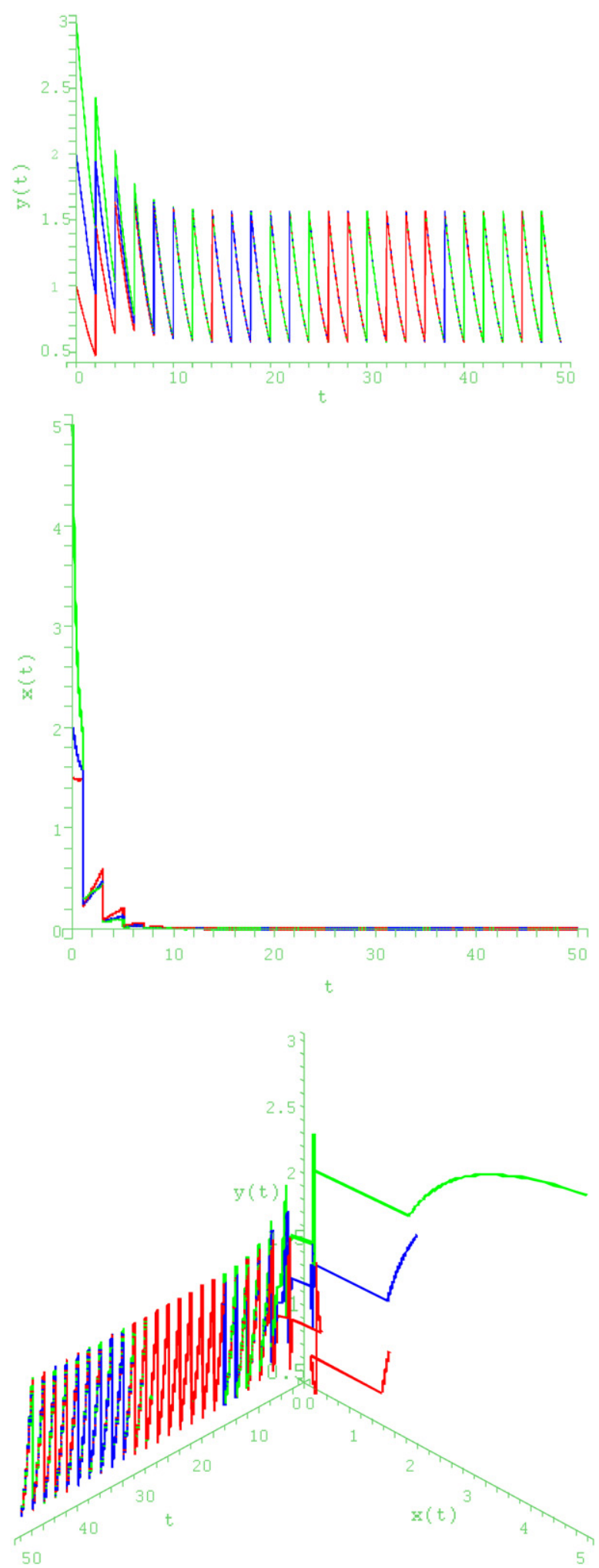

Fig. 2. $\mu=1, p_{2}=0.01, w=0.5, b=1.5, k_{1}=8, k_{2}=0.1, K=1.6, A=2, \tilde{l}=0.5, T=2$. Three trajectories with different initial values approach the periodic trivial orbit of period $T$ at $p_{1}=1-\exp (-1.9)$. 
Let $\alpha=k \bar{\tau} ; k=k(\bar{\tau})$. It follows from the equation above that

$$
f(\bar{\tau})=\bar{\tau}^{2}(\underbrace{\frac{\partial^{2} f}{\partial \bar{\tau} \partial \alpha}(0,0)}_{<0} k+\frac{1}{2} \underbrace{\frac{\partial^{2} f}{\partial \alpha^{2}}(0,0)}_{>0} k^{2}+o(\bar{\tau}, k \bar{\tau})\left(1+k^{2}\right)) .
$$

Obviously, the following equation

$$
\underbrace{\frac{\partial^{2} f}{\partial \bar{\tau} \partial \alpha}(0,0)}_{<0} k+\frac{1}{2} \underbrace{\frac{\partial^{2} f}{\partial \alpha^{2}}(0,0)}_{>0} k^{2}+o(\bar{\tau}, k \bar{\tau})\left(1+k^{2}\right)=0
$$

has a nontrivial positive solution $k=k(\bar{\tau})$ if $\bar{\tau}$ is positive and small enough.

In conclusion, from the above analysis, it is shown that if the necessary condition for bifurcation (15) is satisfied, then a stable positive periodic solution arises via a supercritical bifurcation, so (15) is also sufficient. This discussion may be summarized in the following result.

Theorem 6.1. Assume that condition (15) is satisfied. Then there is $\epsilon>0$ such that for all $0<\bar{\tau}<\epsilon$ there is a stable positive nontrivial periodic solution of (4) with initial data $X_{0}+\alpha(\bar{\tau}) Y_{0}+z(\bar{\tau}, \alpha(\bar{\tau})) E_{0}$ and period $T+\bar{\tau}$.

Remark 6.2. The final part of the existence argument can be pursued by using the substitution $\bar{\tau}=k \alpha ; k=k(\alpha)$ as well.

Remark 6.3. Our bifurcation computations show that there exists a stable positive periodic solution if the impulsive period tends to the solution $T$ of $\tilde{\mathcal{L}}\left(y^{*}(t)\right)=T+\ln \left(1-p_{1}\right)$. The larger the period, the more the pests, so we can control the size of the pest population below some given economically significant levels by choosing an appropriate impulsive period.

\section{Numerical simulations}

To confirm our mathematical findings and facilitate their interpretation, we proceed to investigate further by using numerical simulations (Table 1).
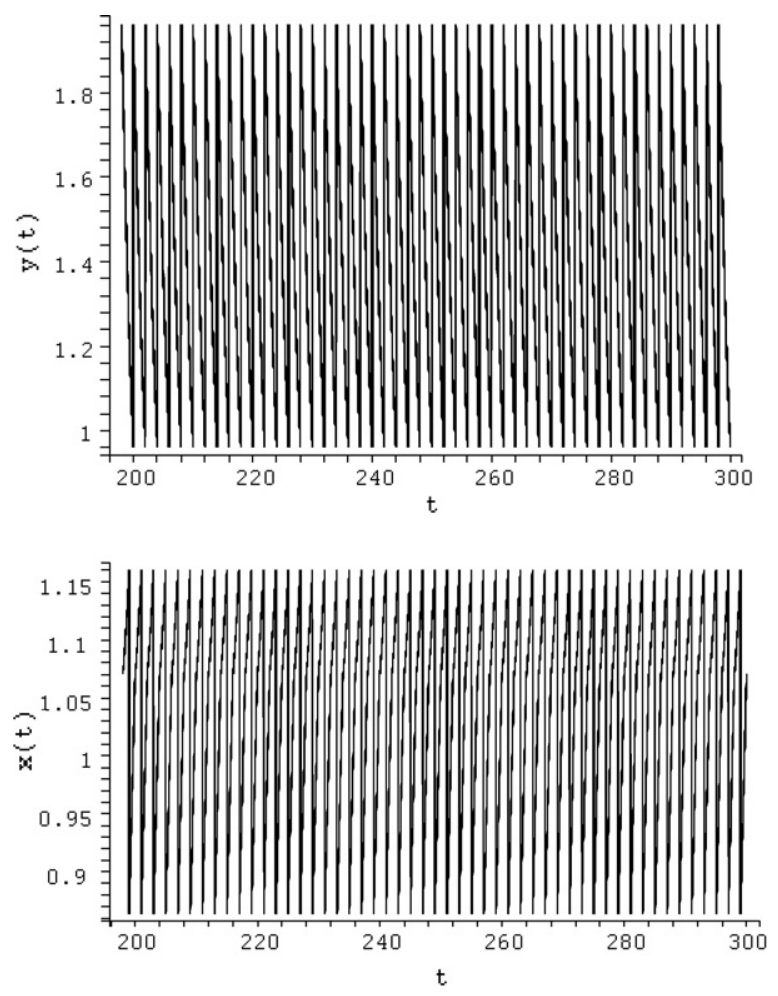

Fig. 3. $\mu=1, p_{2}=0.01, w=0.5, b=1.5, k_{1}=8, k_{2}=0.1, K=1.6, A=2, \tilde{l}=0.5, T=2$ and $x(0)=1.5, y(0)=1$. The time series for $x, y$ indicate that pests and its natural enemies co-exist for $p_{1}=1-\exp (-0.5)$. 


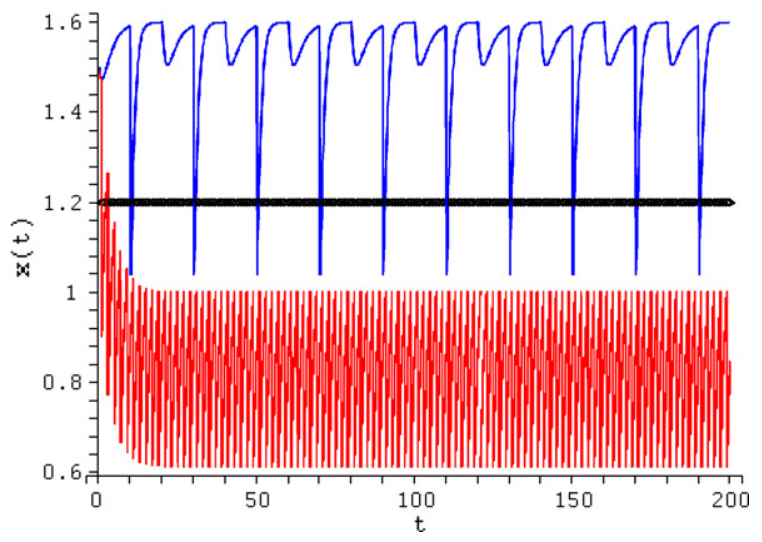

Fig. 4. $\mu=1, p_{2}=0.01, w=0.5, b=1.5, k_{1}=8, k_{2}=0.1, K=1.6, A=2, \tilde{l}=0.5, x(0)=1.5, y(0)=1$ and $p_{1}=1-\exp (-0.5)$. The time series for $x$ at $T=2$ (the red curve) and $T=20$ (the blue curve), respectively. Also $x(t)=1.2$ (the given ET).

We may then answer the crucial questions asked in Section 1. Let $\mu=1, p_{2}=0.01, w=0.5, b=1.5, k_{1}=8, k_{2}=0.1, K=1.6$, $A=2, \tilde{l}=0.5, T=2$ and $x(0)=1.5, y(0)=1$. It is then seen that

$$
\tilde{\mathcal{L}}\left(y^{*}(t)\right)=1.41 \quad \text { and } \quad \mathcal{L}\left(y^{*}(t)\right)=0.199 .
$$

Further, according to Theorem 4.2, we see that for $p_{1}=1-\exp (-0.7)$ and $p_{1}=1-\exp (-1.9)$ the prey (pest)-eradication periodic solution $\left(y^{*}(t), 0\right)$ of the system $(1)$ remains locally stable and globally asymptotically stable, respectively. Indeed, in Fig. 1 we capture a unstable behavior for $\mu=1, p_{2}=0.01, w=0.5, b=1.5, k_{1}=8, k_{2}=0.1, K=1.6, A=2, \tilde{l}=0.5, T=2, p_{1}=1-\exp (-0.7)$ and $x(0)=1.5, y(0)=1$, that is, in this case the trajectory of the corresponding solution of the impulsive system (1) does not tend to the trivial periodic solution and global stability is consequently not achieved. As seen from (6), a suitable increase in the value of $p_{1}$ has the potential to stabilize the behavior of the system globally and make all solutions tend to the prey (pest)-eradication solution $\left(y^{*}, 0\right)$. A typical example of "stable" behavior (global attractor) is captured in Fig. 2 for $\mu=1, p_{2}=0.01, w=0.5, b=1.5$, $k_{1}=8, k_{2}=0.1, K=1.6, A=2, \tilde{l}=0.5, T=2, p_{1}=1-\exp (-1.9)$ and three different initial values $(x(0)=1.5, y(0)=1$; $x(0)=2, y(0)=2$; and $x(0)=5, y(0)=3)$.

For $p_{1}=1-\exp (-0.5)$ and for the same coefficients used to obtain the trajectories pictured in Fig. 2, it then follows from Theorem 5.2 that in this case the pests and their natural enemies ultimately co-exist. The behavior of the trajectory is depicted in Fig. 3.

If we set $p_{1}=1-\exp (-0.5)$ and the economic threshold $E T=1.2$, it is seen that a period $T \leq 8$ stabilizes the pest population $x$ below $E T$, provided that $x(0)=1.5$ and $y(0)=1$. In this regard, Fig. 4 pictures the trajectories of $x$ for $T=2$ and, respectively, for $T=20$.

\section{Conclusion}

In this paper, an integrated pest management model described through an impulsively perturbed predator-prey (pest) system is proposed and investigated. To control the behavior of the system, biological controls, in the form of periodic release of pests' natural enemies in a fixed amount and chemical controls, in the form of periodic pesticide spraying, are employed. It is assumed that the biological and the chemical control are used with the same periodicity, but not simultaneously. It is also assumed that as a result of pesticide spraying fixed proportions of prey (pest) biomass and predator biomass are degraded each time. A Beddington-DeAngelis type function is used to model the functional response of the predator.

By means of the Floquet theory of impulsively perturbed systems of ordinary differential equations and comparison arguments, it is seen that the local stability of the pest-eradication periodic solution is governed by a threshold condition on the net increase of the prey. That is, if $\tilde{\mathcal{L}}\left(y^{*}(t)\right)>T+\ln \left(1-p_{1}\right)$, then the pest (prey)-eradication periodic solution is stable. If the reverse of the condition above is satisfied, then the system is permanent, that is, pests and its natural enemies co-exist forever. It is also seen that this loss of stability is due to the onset of a nontrivial periodic solution which appears via a supercritical bifurcation.

It is also to be noted that in a related prey-dependent model (Georgescu et al., 2008; Zhang et al., 2007a) the condition which ensures the local stability of the trivial periodic solution is actually enough to imply its global stability as well, and this condition displays a significant dependence on the functional response of the predator. In our model, featuring a predator-dependent Beddington-DeAngelis functional response (and probably in any similar predator-dependent model which is not ratio-dependent), the local and global stability conditions, depending on the (per-pest) minimal and, respectively, maximal additional loss of pests in a period $T$ due to predator consumption, are not the same. A sufficient condition for the global stability of the pest-free periodic solution, corresponding to the ultimate success of our pest management strategy, is derived, although, biologically speaking, the inte- 
grated pest management strategy can be considered successful when pest population stabilizes under a certain economic threshold, not necessarily when it is completely removed from the environment.

We note that, theoretically speaking, the control strategy can be always made to succeed by the use of proper pesticides (see Remark 4.3), while as far as the biological control is concerned, its sufficient effectiveness can also be reached provided that the natural enemies is voracious enough ( $b$ is large enough), or the amount $\mu$ of the predator released each time is large enough or the period $T$ is small enough (see again Remark 4.3). Any of these characteristics alone can ensure the success of our control strategy, although, practically speaking, in concrete circumstances they may or may not be feasible.

Finally, we mention some future directions of work extending previous works:

- some situations not detailed in our results which may lead to a chaotic behavior of the system need further study (specifically, the case in which the system is permanent but does not necessarily have a nontrivial periodic solution);

- optimal IPM strategies for impulsive control problem are also worthwhile of study;

- more complicated food chains may also be considered.

\section{Acknowledgments}

The work was supported by the National Natural Science Foundation of China (10771104). The authors are grateful to the referee for useful advice and constructive comments which led to a significant improvement of the presentation.

\section{Appendix A}

\section{A.1. The first order partial derivatives of $f$ at $(0,0)$}

By (16) and (20), it is easy to see that

$$
\begin{aligned}
\frac{\partial f}{\partial \alpha}(\bar{\tau}, \alpha)= & \frac{\partial}{\partial \alpha}\left(\alpha-\Psi_{2}\left(T+\bar{\tau}, X_{0}+\alpha Y_{0}+z(\bar{\tau}, \alpha) E_{0}\right)\right) \\
= & 1-\frac{\partial}{\partial \alpha}\left(\Phi_{2}\left((1-\tilde{l})(T+\bar{\tau}) ; I_{1}\left(\Phi\left(\tilde{l}(T+\bar{\tau}) ; X_{0}+\alpha Y_{0}+z(\bar{\tau}, \alpha) E_{0}\right)\right)\right)\right) \\
= & 1-\frac{\partial \Phi_{2}}{\partial x_{1}}\left((1-\tilde{l})(T+\bar{\tau}) ; I_{1}\left(\Phi\left(\tilde{l}(T+\bar{\tau}) ; X_{0}+\alpha Y_{0}+z(\bar{\tau}, \alpha) E_{0}\right)\right)\right) \times\left(1-p_{2}\right) \\
& \left.\left.\times\left(\frac{\partial \Phi_{1}}{\partial x_{1}} \tilde{l}(T+\bar{\tau}) ; X_{0}+\alpha Y_{0}+z(\bar{\tau}, \alpha) E_{0}\right)\left(-b_{0}^{\prime} / a_{0}^{\prime}+\frac{\partial z}{\partial \alpha}(\bar{\tau}, \alpha)\right)+\frac{\partial \Phi_{1}}{\partial x_{2}} \tilde{l}(T+\bar{\tau}) ; X_{0}+\alpha Y_{0}+z(\bar{\tau}, \alpha) E_{0}\right)\right) \\
& -\frac{\partial \Phi_{2}}{\partial x_{2}}\left((1-\tilde{l})(T+\bar{\tau}) ; I_{1}\left(\Phi\left(\tilde{l}(T+\bar{\tau}) ; X_{0}+\alpha Y_{0}+z(\bar{\tau}, \alpha) E_{0}\right)\right)\right) \times\left(1-p_{1}\right) \\
& \left.\left.\times\left(\frac{\partial \Phi_{2}}{\partial x_{1}} \tilde{l}(T+\bar{\tau}) ; X_{0}+\alpha Y_{0}+z(\bar{\tau}, \alpha) E_{0}\right)\left(-b_{0}^{\prime} / a_{0}^{\prime}+\frac{\partial z}{\partial \alpha}(\bar{\tau}, \alpha)\right)+\frac{\partial \Phi_{2}}{\partial x_{2}} \tilde{l}(T+\bar{\tau}) ; X_{0}+\alpha Y_{0}+z(\bar{\tau}, \alpha) E_{0}\right)\right) .
\end{aligned}
$$

Combining the above with (17), it follows that

$$
\left.\frac{\partial f}{\partial \alpha}(0,0)=1-\frac{\partial \Phi_{2}}{\partial x_{2}}\left((1-\tilde{l}) T ; I_{1}\left(\Phi\left(\tilde{l} T ; X_{0}\right)\right)\right)\left(1-p_{1}\right) \frac{\partial \Phi_{2}}{\partial x_{2}} \tilde{l} T ; X_{0}\right)=d_{0}^{\prime}=0 .
$$

One may deduce that

$$
\begin{aligned}
\frac{\partial f}{\partial \bar{\tau}}(\bar{\tau}, \alpha)= & \frac{\partial}{\partial \bar{\tau}}\left(\alpha-\Psi_{2}\left(T+\bar{\tau}, X_{0}+\alpha Y_{0}+z(\bar{\tau}, \alpha) E_{0}\right)\right) \\
= & -\frac{\partial}{\partial \bar{\tau}}\left(\Phi_{2}\left((1-\tilde{l})(T+\bar{\tau}) ; I_{1}\left(\Phi\left(\tilde{l}(T+\bar{\tau}) ; X_{0}+\alpha Y_{0}+z(\bar{\tau}, \alpha) E_{0}\right)\right)\right)\right) \\
= & -\frac{\partial \Phi_{2}}{\partial \bar{\tau}}\left((1-\tilde{l})(T+\bar{\tau}) ; I_{1}\left(\Phi\left(\tilde{l}(T+\bar{\tau}) ; X_{0}+\alpha Y_{0}+z(\bar{\tau}, \alpha) E_{0}\right)\right)\right)(1-\tilde{l}) \\
& -\frac{\partial \Phi_{2}}{\partial x_{1}}\left((1-\tilde{l})(T+\bar{\tau}) ; I_{1}\left(\Phi\left(\tilde{l}(T+\bar{\tau}) ; X_{0}+\alpha Y_{0}+z(\bar{\tau}, \alpha) E_{0}\right)\right)\right)
\end{aligned}
$$




$$
\begin{aligned}
& \times\left(1-p_{2}\right)\left(\frac{\partial \Phi_{1}}{\partial \bar{\tau}}\left(\tilde{l}(T+\bar{\tau}) ; X_{0}+\alpha Y_{0}+z(\bar{\tau}, \alpha) E_{0}\right) \tilde{l}+\frac{\partial \Phi_{1}}{\partial x_{1}}\left(\tilde{l}(T+\bar{\tau}) ; X_{0}+\alpha Y_{0}+z(\bar{\tau}, \alpha) E_{0}\right) \frac{\partial z}{\partial \bar{\tau}}(\bar{\tau}, \alpha)\right) \\
& -\frac{\partial \Phi_{2}}{\partial x_{2}}\left((1-\tilde{l})(T+\bar{\tau}) ; I_{1}\left(\Phi\left(\tilde{l}(T+\bar{\tau}) ; X_{0}+\alpha Y_{0}+z(\bar{\tau}, \alpha) E_{0}\right)\right)\right) \times\left(1-p_{1}\right) \\
& \times\left(\frac{\partial \Phi_{2}}{\partial \bar{\tau}}\left(\tilde{l}(T+\bar{\tau}) ; X_{0}+\alpha Y_{0}+z(\bar{\tau}, \alpha) E_{0}\right) \tilde{l}+\frac{\partial \Phi_{2}}{\partial x_{1}}\left(\tilde{l}(T+\bar{\tau}) ; X_{0}+\alpha Y_{0}+z(\bar{\tau}, \alpha) E_{0}\right) \frac{\partial z}{\partial \bar{\tau}}(\bar{\tau}, \alpha)\right) .
\end{aligned}
$$

Since (21) holds and

$$
\begin{aligned}
& \frac{\partial \Phi_{2}}{\partial x_{1}}\left((1-\tilde{l}) T ; I_{1}\left(\Phi\left(\tilde{l} T ; X_{0}\right)\right)\right)=0 \\
& \frac{\partial \Phi_{2}}{\partial \bar{\tau}}\left((1-\tilde{l}) T ; I_{1}\left(\Phi\left(\tilde{l} T ; X_{0}\right)\right)\right)=0
\end{aligned}
$$

it consequently follows that

$$
\frac{\partial f}{\partial \bar{\tau}}(0,0)=0 \text {. }
$$

\section{A.2. The second-order partial derivatives of $f$ at $(0,0)$}

Before computing the above-mentioned partial derivatives, we need to know $\left(\partial^{2} \Phi_{2} / \partial x_{1}^{2}\right),\left(\partial^{2} \Phi_{2} / \partial x_{2}^{2}\right)$ and $\left(\partial^{2} \Phi_{2} / \partial x_{1} \partial x_{2}\right)$. By formally deriving

$$
\frac{\mathrm{d}}{\mathrm{d} t}\left(\Phi\left(t ; X_{0}\right)\right)=F\left(\Phi\left(t ; X_{0}\right)\right)
$$

one sees that

$$
\frac{\partial \Phi_{2}}{\partial x_{1}}\left(t ; X_{0}\right)=\mathrm{e}^{\int_{0}^{t}\left(1-\mathcal{P}\left(\Phi_{1}\left(s ; X_{0}\right)\right)\right) \mathrm{d} s} \frac{\partial \Phi_{2}}{\partial x_{1}}\left(0 ; X_{0}\right),
$$

which yields that $\left(\partial \Phi_{2} / \partial x_{1}\right)\left(t ; X_{0}\right) \equiv 0$, since $\left(\partial \Phi_{2} / \partial x_{1}\right)\left(0 ; X_{0}\right)=0$. Also

$$
\begin{gathered}
\frac{\partial \Phi_{1}}{\partial x_{1}}\left(t ; X_{0}\right)=\mathrm{e}^{-w t} \\
\frac{\partial \Phi_{2}}{\partial x_{2}}\left(t ; X_{0}\right)=\mathrm{e}^{\int_{0}^{t}\left(1-\mathcal{P}\left(\Phi_{1}\left(s ; X_{0}\right)\right)\right) \mathrm{d} s} \frac{\partial \Phi_{2}}{\partial x_{2}}\left(0 ; X_{0}\right)=\mathrm{e}^{\int_{0}^{t}\left(1-\mathcal{P}\left(\Phi_{1}\left(s ; X_{0}\right)\right)\right) \mathrm{d} s} ; \\
\frac{\partial \Phi_{1}}{\partial x_{2}}\left(t ; X_{0}\right)=\mathrm{e}^{-w t} \int_{0}^{t} \mathcal{P}\left(\Phi_{1}\left(s ; X_{0}\right)\right) \mathrm{e}^{\int_{0}^{s}\left[(1+w)-\mathcal{P}\left(\Phi_{1}\left(\tau ; X_{0}\right)\right)\right] \mathrm{d} \tau} \mathrm{d} s
\end{gathered}
$$

In view of $\left(\partial \Phi_{2} / \partial x_{1}\right)\left(0 ; X_{0}\right)=0$ and $\left(\partial^{2} \Phi_{2} / \partial^{2} x_{1}\right)\left(0 ; X_{0}\right)=0$, then

$$
\begin{aligned}
& \frac{\partial^{2} \Phi_{2}}{\partial x_{1}^{2}}\left(t, X_{0}\right)=\mathrm{e}_{0}^{t}\left(1-\mathcal{P}\left(\Phi_{1}\left(s ; X_{0}\right)\right)\right) \mathrm{d} s \frac{\partial^{2} \Phi_{2}}{\partial^{2} x_{1}}\left(0 ; X_{0}\right)=0, \\
& \frac{\partial^{2} \Phi_{2}}{\partial x_{2}^{2}}\left(t, X_{0}\right)=-\frac{\partial \Phi_{2}}{\partial x_{2}}\left(t ; X_{0}\right) \int_{0}^{t} \mathcal{P}^{\prime}\left(\Phi_{1}\left(s ; X_{0}\right)\right) \frac{\partial \Phi_{1}}{\partial x_{2}}\left(s ; X_{0}\right) \mathrm{d} s,
\end{aligned}
$$

and

$$
\frac{\partial^{2} \Phi_{2}}{\partial x_{2} \partial x_{1}}\left(t, X_{0}\right)=-\frac{\partial \Phi_{2}}{\partial x_{2}}\left(t ; X_{0}\right) \int_{0}^{t} \mathcal{P}^{\prime}\left(\Phi_{1}\left(s ; X_{0}\right)\right) \frac{\partial \Phi_{1}}{\partial x_{1}}\left(s ; X_{0}\right) \mathrm{d} s .
$$

One remarks that

$$
\frac{\partial^{2} \Phi_{2}}{\partial x_{1} \partial \bar{\tau}}\left((1-\tilde{l}) T ; I_{1}\left(\Phi\left(\tilde{l} T ; X_{0}\right)\right)\right)=0
$$




$$
\begin{aligned}
& \frac{\partial^{2} \Phi_{2}}{\partial x_{1}^{2}}\left((1-\tilde{l}) T ; I_{1}\left(\Phi\left(\tilde{l} T ; X_{0}\right)\right)\right)=0 \\
& \frac{\partial^{2} \Phi_{2}}{\partial x_{1}^{2}}\left(\tilde{l} T ; X_{0}\right)=0
\end{aligned}
$$

From (30) and (33)-(35) together with $z(0,0)=0$, we get that

$$
\frac{\partial^{2} f}{\partial \bar{\tau}^{2}}(0,0)=-\frac{\partial^{2} \Phi_{2}}{\partial \bar{\tau}^{2}}\left((1-\tilde{l}) T ; I_{1}\left(\Phi\left(\tilde{l} T ; X_{0}\right)\right)\right)(1-\tilde{l})^{2} .
$$

Since

$$
\frac{\partial^{2} \Phi}{\partial \bar{\tau}^{2}}\left((1-\tilde{l}) T ; I_{1}\left(\Phi\left(\tilde{l} T ; X_{0}\right)\right)\right)=0,
$$

it is then concluded that

$$
\frac{\partial^{2} f}{\partial \bar{\tau}^{2}}(0,0)=0
$$

According to (29), one notes that

$$
\begin{aligned}
\frac{\partial^{2} f}{\partial \alpha^{2}}(\bar{\tau}, \alpha)= & \frac{\partial^{2}}{\partial \alpha^{2}}\left(\alpha-\Psi_{2}\left(T+\bar{\tau}, X_{0}+\alpha Y_{0}+z(\bar{\tau}, \alpha) E_{0}\right)\right) \\
= & -\frac{\partial^{2}}{\partial \alpha^{2}}\left(\Phi_{2}\left((1-\tilde{l})(T+\bar{\tau}) ; I_{1}\left(\Phi\left(\tilde{l}(T+\bar{\tau}) ; X_{0}+\alpha Y_{0}+z(\bar{\tau}, \alpha) E_{0}\right)\right)\right)\right) \\
= & -\frac{\partial}{\partial \alpha}\left[\frac{\partial \Phi_{2}}{\partial x_{1}}\left((1-\tilde{l})(T+\bar{\tau}) ; I_{1}\left(\Phi\left(\tilde{l}(T+\bar{\tau}) ; X_{0}+\alpha Y_{0}+z(\bar{\tau}, \alpha) E_{0}\right)\right)\right)\right] \\
& \times\left(1-p_{2}\right)\left(\frac{\partial \Phi_{1}}{\partial x_{1}}\left(\tilde{l}(T+\bar{\tau}) ; X_{0}+\alpha Y_{0}+z(\bar{\tau}, \alpha) E_{0}\right)\left(-b_{0}^{\prime} / a_{0}^{\prime}+\frac{\partial z}{\partial \alpha}(\bar{\tau}, \alpha)\right)+\frac{\partial \Phi_{1}}{\partial x_{2}}\left(\tilde{l}(T+\bar{\tau}) ; X_{0}+\alpha Y_{0}+z(\bar{\tau}, \alpha) E_{0}\right)\right) \\
& -\frac{\partial \Phi_{2}}{\partial x_{1}}\left((1-\tilde{l})(T+\bar{\tau}) ; I_{1}\left(\Phi\left(\tilde{l}(T+\bar{\tau}) ; X_{0}+\alpha Y_{0}+z(\bar{\tau}, \alpha) E_{0}\right)\right)\right)\left(1-p_{2}\right) \\
& \times \frac{\partial}{\partial \alpha}\left[\left(\frac{\partial \Phi_{1}}{\partial x_{1}}\left(\tilde{l}(T+\bar{\tau}) ; X_{0}+\alpha Y_{0}+z(\bar{\tau}, \alpha) E_{0}\right)\left(-b_{0}^{\prime} / a_{0}^{\prime}+\frac{\partial z}{\partial \alpha}(\bar{\tau}, \alpha)\right)+\frac{\partial \Phi_{1}}{\partial x_{2}}\left(\tilde{l}(T+\bar{\tau}) ; X_{0}+\alpha Y_{0}+z(\bar{\tau}, \alpha) E_{0}\right)\right)\right] \\
& -\frac{\partial}{\partial \alpha}\left[\frac{\partial \Phi_{2}}{\partial x_{2}}\left((1-\tilde{l})(T+\bar{\tau}) ; I_{1}\left(\Phi\left(\tilde{l}(T+\bar{\tau}) ; X_{0}+\alpha Y_{0}+z(\bar{\tau}, \alpha) E_{0}\right)\right)\right)\right] \\
& \times\left(1-p_{1}\right)\left(\frac{\partial \Phi_{1}}{\partial x_{1}}\left(\tilde{l}(T+\bar{\tau}) ; X_{0}+\alpha Y_{0}+z(\bar{\tau}, \alpha) E_{0}\right)\left(-b_{0}^{\prime} / a_{0}^{\prime}+\frac{\partial z}{\partial \alpha}(\bar{\tau}, \alpha)\right)+\frac{\partial \Phi_{1}}{\partial x_{2}}\left(\tilde{l}(T+\bar{\tau}) ; X_{0}+\alpha Y_{0}+z(\bar{\tau}, \alpha) E_{0}\right)\right) \\
& -\frac{\partial \Phi_{2}}{\partial x_{2}}\left((1-\tilde{l})(T+\bar{\tau}) ; I_{1}\left(\Phi\left(\tilde{l}(T+\bar{\tau}) ; X_{0}+\alpha Y_{0}+z(\bar{\tau}, \alpha) E_{0}\right)\right)\right)\left(1-p_{1}\right) \\
& \times \frac{\partial}{\partial \alpha}\left[\left(\frac{\partial \Phi_{2}}{\partial x_{1}}\left(\tilde{l}(T+\bar{\tau}) ; X_{0}+\alpha Y_{0}+z(\bar{\tau}, \alpha) E_{0}\right)\left(-b_{0}^{\prime} / a_{0}^{\prime}+\frac{\partial z}{\partial \alpha}(\bar{\tau}, \alpha)\right)+\frac{\partial \Phi_{2}}{\partial x_{2}}\left(\tilde{l}(T+\bar{\tau}) ; X_{0}+\alpha Y_{0}+z(\bar{\tau}, \alpha) E_{0}\right)\right)\right]
\end{aligned}
$$

Then

$$
\begin{aligned}
\frac{\partial^{2} f}{\partial \alpha^{2}}(0,0)= & -\frac{\partial}{\partial \alpha}\left[\frac{\partial \Phi_{2}}{\partial x_{1}}\left((1-\tilde{l})(T+\bar{\tau}) ; I_{1}\left(\Phi\left(\tilde{l}(T+\bar{\tau}) ; X_{0}+\alpha Y_{0}+z(\bar{\tau}, \alpha) E_{0}\right)\right)\right)\right]_{(\bar{\tau}, \alpha)=(0,0)} \\
& \times\left(1-p_{2}\right)\left(\frac{\partial \Phi_{1}}{\partial x_{1}}\left(\tilde{l} T ; X_{0}\right)\left(-b_{0}^{\prime} / a_{0}^{\prime}+\frac{\partial z}{\partial \alpha}(\bar{\tau}, \alpha)\right)+\frac{\partial \Phi_{1}}{\partial x_{2}}\left(\tilde{l} T ; X_{0}\right)\right) \\
& -\frac{\partial}{\partial \alpha}\left[\frac{\partial \Phi_{2}}{\partial x_{2}}\left((1-\tilde{l})(T+\bar{\tau}) ; I_{1}\left(\Phi\left(\tilde{l}(T+\bar{\tau}) ; X_{0}+\alpha Y_{0}+z(\bar{\tau}, \alpha) E_{0}\right)\right)\right)\right]_{(\bar{\tau}, \alpha)=(0,0)} \\
& \times\left(1-p_{1}\right)\left(\frac{\partial \Phi_{2}}{\partial x_{1}}\left(\tilde{l} T ; X_{0}\right)\left(-b_{0}^{\prime} / a_{0}^{\prime}\right)+\frac{\partial \Phi_{2}}{\partial x_{2}}\left(\tilde{l} T ; X_{0}\right)\right)-\frac{\partial \Phi_{2}}{\partial x_{2}}\left((1-\tilde{l}) T ; I_{1}\left(\Phi\left(\tilde{l} T ; X_{0}\right)\right)\right)\left(1-p_{1}\right)
\end{aligned}
$$




$$
\begin{aligned}
& \times \frac{\partial}{\partial \alpha}\left[\left(\frac{\partial \Phi_{2}}{\partial x_{1}}\left(\tilde{l}(T+\bar{\tau}) ; X_{0}+\alpha Y_{0}+z(\bar{\tau}, \alpha) E_{0}\right)\left(-b_{0}^{\prime} / a_{0}^{\prime}+\frac{\partial z}{\partial \alpha}(\bar{\tau}, \alpha)\right)\right.\right. \\
& \left.\left.+\frac{\partial \Phi_{2}}{\partial x_{2}}\left(\tilde{l}(T+\bar{\tau}) ; X_{0}+\alpha Y_{0}+z(\bar{\tau}, \alpha) E_{0}\right)\right)\right]_{(\bar{\tau}, \alpha)=(0,0)} .
\end{aligned}
$$

After a few computations, we derive that

$$
\begin{aligned}
\frac{\partial^{2} f}{\partial \alpha^{2}}(0,0)= & -\left(\frac{\partial^{2} \Phi_{2}}{\partial x_{1} \partial x_{2}}\left((1-\tilde{l}) T ; I_{1}\left(\Phi\left(\tilde{l} T ; X_{0}\right)\right)\right)+\frac{\partial^{2} \Phi_{2}}{\partial x_{2} \partial x_{1}}\left((1-\tilde{l}) T ; I_{1}\left(\Phi\left(\tilde{l} T ; X_{0}\right)\right)\right)\right) \\
& \times\left(1-p_{1}\right)\left(1-p_{2}\right)\left(\frac{\partial \Phi_{1}}{\partial x_{1}}\left(\tilde{l} T ; X_{0}\right)\left(-b_{0}^{\prime} / a_{0}^{\prime}\right)+\frac{\partial \Phi_{1}}{\partial x_{2}}\left(\tilde{l} T ; X_{0}\right)\right) \frac{\partial \Phi_{2}}{\partial x_{2}}\left(\tilde{l} T ; X_{0}\right) \\
& -\frac{\partial^{2} \Phi_{2}}{\partial x_{2}^{2}}\left((1-\tilde{l}) T ; I_{1}\left(\Phi\left(\tilde{l} T ; X_{0}\right)\right)\right)\left(1-p_{1}\right)^{2}\left(\frac{\partial \Phi_{2}}{\partial x_{2}}\left(\tilde{l} T ; X_{0}\right)\right)^{2}-\frac{\partial \Phi_{2}}{\partial x_{2}}\left((1-\tilde{l}) T ; I_{1}\left(\Phi\left(\tilde{l} T ; X_{0}\right)\right)\right)\left(1-p_{1}\right) \\
& \left.\times\left[\left(\frac{\partial^{2} \Phi_{2}}{\partial x_{1} \partial x_{2}} \tilde{l} T ; X_{0}\right)+\frac{\partial^{2} \Phi_{2}}{\partial x_{2} \partial x_{1}}\left(\tilde{l} T ; X_{0}\right)\right)\left(-b_{0}^{\prime} / a_{0}^{\prime}\right)+\frac{\partial^{2} \Phi_{2}}{\partial x_{2}^{2}}\left(\tilde{l} T ; X_{0}\right)\right] .
\end{aligned}
$$

Obviously, it can be deduced from the above that

$$
\frac{\partial^{2} f}{\partial \alpha^{2}}(0,0)>0 .
$$

It also seen that

$$
\begin{aligned}
\frac{\partial^{2} f}{\partial \bar{\tau} \partial \alpha}(0,0)= & -\frac{\partial^{2} \Phi_{2}}{\partial x_{2} \partial \bar{\tau}}\left((1-\tilde{l}) T ; I_{1}\left(\Phi\left(\tilde{l} T ; X_{0}\right)\right)\right)\left(1-p_{1}\right) \frac{\partial \Phi_{2}}{\partial x_{2}}\left(\tilde{l} T ; X_{0}\right)(1-\tilde{l}) \\
& -\frac{\partial^{2} \Phi_{2}}{\partial x_{2} \partial x_{1}}\left((1-\tilde{l}) T ; I_{1}\left(\Phi\left(\tilde{l} T ; X_{0}\right)\right)\right)\left(1-p_{1}\right) \frac{\partial \Phi_{2}}{\partial x_{2}}\left(\tilde{l} T ; X_{0}\right)\left(1-p_{2}\right)\left(\frac{\partial \Phi_{1}}{\partial \bar{\tau}}\left(\tilde{l} T ; X_{0}\right) \tilde{l}+\frac{\partial \Phi_{1}}{\partial x_{1}}\left(\tilde{l} T ; X_{0}\right) \frac{\partial z}{\partial \bar{\tau}}(0,0)\right) \\
& -\frac{\partial \Phi_{2}}{\partial x_{2}}\left((1-\tilde{l}) T ; I_{1}\left(\Phi\left(\tilde{l} T ; X_{0}\right)\right)\right)\left(1-p_{1}\right)\left(\frac{\partial^{2} \Phi_{2}}{\partial x_{2} \partial \bar{\tau}}\left(\tilde{l} T ; X_{0}\right) \tilde{l}+\frac{\partial^{2} \Phi_{2}}{\partial x_{2} \partial x_{1}}\left(\tilde{l} T ; X_{0}\right) \frac{\partial z}{\partial \bar{\tau}}(0,0)\right) .
\end{aligned}
$$

Next, we compute the right-hand side of the equation above. It is seen that

$$
\begin{aligned}
& -\frac{\partial^{2} \Phi_{2}}{\partial x_{2} \partial \bar{\tau}}\left((1-\tilde{l}) T ; I_{1}\left(\Phi\left(\tilde{l} T ; X_{0}\right)\right)\right)\left(1-p_{1}\right) \frac{\partial \Phi_{2}}{\partial x_{2}}\left(\tilde{l} T ; X_{0}\right)(1-\tilde{l})=-\left(1-\mathcal{P}\left(y^{*}(T)\right)\right)(1-\tilde{l}) ; \\
& -\frac{\partial^{2} \Phi_{2}}{\partial x_{2} \partial x_{1}}\left((1-\tilde{l}) T ; I_{1}\left(\Phi\left(\tilde{l} T ; X_{0}\right)\right)\right)\left(1-p_{1}\right) \frac{\partial \Phi_{2}}{\partial x_{2}}\left(\tilde{l} T ; X_{0}\right)=\int_{0}^{(1-\tilde{l}) T} \mathcal{P}^{\prime}\left(y^{*}(s+\tilde{l} T)\right) e^{-w s} \mathrm{~d} s ; \\
& \left(1-p_{2}\right)\left(\frac{\partial \Phi_{1}}{\partial \bar{\tau}}\left(\tilde{l} T ; X_{0}\right) \tilde{l}+\frac{\partial \Phi_{1}}{\partial x_{1}}\left(\tilde{l} T ; X_{0}\right) \frac{\partial z}{\partial \bar{\tau}}(0,0)\right)=\left(1-p_{2}\right)\left(-w y^{*}(\tilde{l} T) \tilde{l}-\frac{\mathrm{e}^{-w \tilde{l} T} w y^{*}(T)}{a_{0}^{\prime}}\right) ; \\
& \left.-\frac{\partial \Phi_{2}}{\partial x_{2}}\left((1-\tilde{l}) T ; I_{1}\left(\Phi\left(\tilde{l} T ; X_{0}\right)\right)\right)\left(1-p_{1}\right)\left(\frac{\partial^{2} \Phi_{2}}{\partial x_{2} \partial \tilde{\tau}}\left(\tilde{l} T ; X_{0}\right) \tilde{l}+\frac{\partial^{2} \Phi_{2}}{\partial x_{2} \partial x_{1}} \tilde{l} T ; X_{0}\right) \frac{\partial z}{\partial \bar{\tau}}(0,0)\right) \\
& =-\frac{\partial \Phi_{2}}{\partial x_{2}}\left((1-\tilde{l}) T ; I_{1}\left(\Phi\left(\tilde{l} T ; X_{0}\right)\right)\right)\left(1-p_{1}\right) \\
& \times\left[\left(1-\mathcal{P}\left(y^{*}(\tilde{l} T)\right)\right) \frac{\partial \Phi_{2}}{\partial x_{2}}\left(\tilde{l} T ; X_{0}\right) \tilde{l}-\left(\frac{\partial \Phi_{2}}{\partial x_{2}}\left(\tilde{l} T ; X_{0}\right) \int_{0}^{\tilde{l} T} \mathcal{P}^{\prime}\left(y^{*}(s)\right) \mathrm{e}^{-w s} \mathrm{~d} s\right) \frac{\partial z}{\partial \bar{\tau}}(0,0)\right] \\
& =-\left[\left(1-\mathcal{P}\left(y^{*}(\tilde{l} T)\right)\right) \tilde{l}+\int_{0}^{\tilde{l} T} \mathcal{P}^{\prime}\left(y^{*}(s)\right) \mathrm{e}^{-w s} \mathrm{~d} s \frac{w y^{*}(T)}{a_{0}^{\prime}}\right]
\end{aligned}
$$


Hence, it is concluded that

$$
\begin{aligned}
\frac{\partial^{2} f}{\partial \bar{\tau} \partial \alpha}(0,0)= & -\left[1-\tilde{l} \mathcal{P}\left(y^{*}(\tilde{l} T)\right)-(1-\tilde{l}) \mathcal{P}\left(y^{*}(T)\right)\right]-w\left(\int_{\tilde{l} T}^{T} \mathcal{P}^{\prime}\left(y^{*}(s)\right) \mathrm{e}^{-w s} \mathrm{~d} s\right)\left(1-p_{2}\right)\left(y^{*}\left(0^{+}\right) \tilde{l}+\frac{y^{*}(T)}{a_{0}^{\prime}}\right) \\
& -\left(\int_{0}^{\tilde{l} T} \mathcal{P}^{\prime}\left(y^{*}(s)\right) \mathrm{e}^{-w s} \mathrm{~d} s\right) \frac{w y^{*}(T)}{a_{0}^{\prime}} .
\end{aligned}
$$

Due to the limiting condition (15), and also, since $y^{*}(\cdot)$ is decreasing on $(0, T]$ and $\mathcal{P}(\cdot)$ is increasing with respect to $y^{*}$, it is easy to derive that

$$
\tilde{l} \mathcal{P}\left(y^{*}(\tilde{l} T)\right)+(1-\tilde{l}) \mathcal{P}\left(y^{*}(T)\right)<\frac{1}{T}\left[\int_{0}^{\tilde{l} T} \mathcal{P}\left(y^{*}(s)\right) \mathrm{d} s+\int_{\tilde{l} T}^{T} \mathcal{P}\left(y^{*}(s)\right) \mathrm{d} s\right]=\frac{1}{T} \int_{0}^{T} \mathcal{P}\left(y^{*}(s)\right) \mathrm{d} s=1+\frac{1}{T} \ln \left(1-p_{1}\right) .
$$

Consequently, one notes that

$$
\frac{\partial^{2} f}{\partial \bar{\tau} \partial \alpha}(0,0)<0 .
$$

\section{References}

Abrams, A., Ginzburg, L.R., 2000. The nature of predation: prey dependent, ratio dependent or neither? Trends Ecol. Evol. 15, $337-341$.

Arditi, R., Ginzburg, L.R., 1989. Coupling in predator-prey dynamics: ratio-dependence. J. Theor. Biol. 139, 311-326.

Bainov, D.D., Simeonov, P.S., 1993. Impulsive Differential Equations: Periodic Solutions and Applications. Longman, London.

Beddington, J.R., 1975. Mutual interference between parasites or predators and its effect on searching efficiency. J. Anim. Ecol. 44, 331-340.

Chow, S.N., Hale, J., 1982. Methods of bifurcation theory. Springer Verlag, New York.

DeAngelis, D.L., Goldstein, R.A., O’Neill, R.V., 1975. A model for tropic interaction. Ecology 56, 881-892.

Debach, P., Rosen, D., 1991. Biological Control by Natural Enemies, 2nd ed. Cambridge University Press, Cambridge.

Ferron, P., 1981. Pest Control Using the Fungi Beauveria and Metarhizinm. In: Burges, H.D. (Ed.), Microbial Control in Pests and Plant Diseases. Academic Press, London.

Freedman, H.J., 1976. Graphical stability, enrichment, and pest control by a natural enemy. Math. Biosci. 31, 207-225.

Georgescu, P., Zhang, H., Chen, L.S., 2008. Bifurcation of nontrivial periodic solutions for an impulsively controlled pest management model. Appl. Math. Comput., doi:10.1016/j.amc.2008.03.012.

Gutierrez, A.P., 1992. The physiological basis of ratio-dependent predator-prey theory: a metabolic pool model of Nicholsons blowflies as an example. Ecology 73, $1552-1563$.

Huisman, C., DeBoer, R.J., 1997. A formal derivation of the Beddington functional response. J. Theor. Biol. 185, 389-400.

Jost, C., Ellner, S., 2000. Testing for predator dependence in predator-prey dynamics: a nonparametric approach. Proc. Roy. Soc. Lond. B 267, 1611-1620.

Kuang, Y., Beretta, E., 1998. Global qualitative analysis of a ratio-dependent predator-prey system. J. Math. Biol. 36, 389-406.

Lakmeche, A., Arino, O., 2000. Bifurcation of non trivial periodic solutions of impulsive differential equations arising chemotherapeutic treatment. Dyn. Cont., Discrete Impuls. Syst. 7, 265-287.

Lakshmikantham, V., Bainov, D.D., Simeonov, P.S., 1989. Theory of Impulsive Differential Equations. World Scientific, Singapore.

Liu, B., Chen, L.S., Zhang, Y.J., 2005. The dynamics of a prey-dependent consumption model concerning impulsive control strategy. Appl. Math. Comp. 169, 305-320.

Liu, B., Zhi, Y., Chen, L.S., 2004. The dynamics of a predator-prey model with Ivlevs functional response concerning integrated pest management. Acta Math. Appl. Sin. Engl. 20, 133-146.

Liu, S., Beretta, E., 2006. A stage-structured predator-prey model of Beddington-DeAngelis type. SIAM J. Appl. Math. 66, 1101-1129.

Luff, M.L., 1983. The potential of predators for pest control. Agric. Ecos. Environ. 10, 159-181.

Skalski, G.T., Gilliam, J.F., 2001. Functional responses with predator interference: viable alternatives to the Holling type II model. Ecology 82, $3083-3092$.

Stern, V.M., Smith, R.F., Van den Bosch, R., Hagen, K.S., 1959. The integrated control concept. Hilgardia 29, 81-101.

Tang, S.Y., Chen, L.S., 2004. Modelling and analysis of integrated pest management strategy. Discrete Cont. Dyn. Syst. B 4, 759-768.

Van den Bosch, R., 1978. The Pesticide Conspiracy. Doubleday \& Co, Garden City, New York.

Zhang, H., Chen, L.S., Georgescu, P., 2007a. Impulsive control strategies for pest management. J. Biol. Syst. 15, 235-260.

Zhang, H., Jiao, J.J., Chen, L.S., 2007b. Pest management through continuous and impulsive control strategies. BioSystems 90, 350-361.

Zhang, Y., Liu, B., Chen, L.S., 2004. Dynamical behavior of Volterra model with mutual interference concerning IPM. ESAIM Math. Mod. Numer. Anal. 38, $143-155$. 